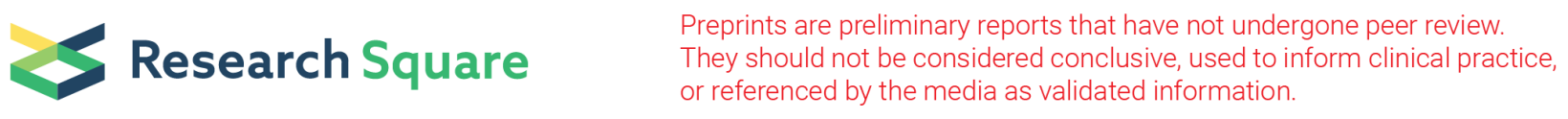

\title{
The characteristics and effectiveness of pregnancy yoga interventions: A systematic review and meta-analysis
}

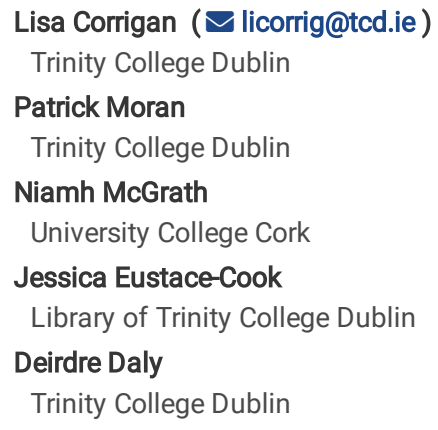

Version of Record: A version of this preprint was published at BMC Pregnancy and Childbirth on March 25th, 2022. See the published version at https://doi.org/10.1186/s12884-022-04474-9. 


\section{Abstract}

Background: Yoga is a popular mind-body medicine frequently recommended and to pregnant women. Gaps remain in our understanding of core components of effective pregnancy yoga programmes. This review and meta-analysis examined the characteristics and effectiveness of pregnancy yoga, incorporating the FITT (frequency, intensity, time/duration and type) principle of exercise prescription in the analysis.

Methods: The following electronic databases were searched: MEDLINE, PsycINFO, EMBASE, CINAHL, WHOLiS, AMED, ScieLo, ASSIA and Web of Science. Randomised control trials and quasi-experimental studies examining pregnancy yoga interventions were eligible for inclusion. Covidence was used to screen titles, abstracts, and full text articles. Outcomes of interest were stress, anxiety, depression, quality of life, labour duration, pain management in labour and mode of birth. The Cochrane Collaboration's Risk of Bias Assessment tool was used to assess methodological quality of studies and GRADE criteria (Gradepro) evaluated quality of the evidence. Meta-analysis was performed using Revman 5.3.

Results: 678 citations were retrieved, 24 studies met inclusion criteria. 22 studies with 1826 pregnant women were included for meta-analysis. Applying perprotocol analysis and a random effects model, the pooled standardised mean difference (SMD) for depression $(-0.53 ; 95 \% \mathrm{Cl}:-1.04$ to -0.02 , $\mathrm{P}=0.04)$, anxiety $(-0.82 ; 95 \% \mathrm{Cl}:-1.64$ to $-0.01 ; \mathrm{p}=0.05)$, perceived stress $(-1.03 ; 95 \% \mathrm{Cl}:-1.55$ to $-0.52 ; \mathrm{p}<0.0001)$ and physiological stress $(-0.24 ; 95 \% \mathrm{Cl}:-0.52$ to $0.04, \mathrm{P}=0.09)$ supported a statistically significant beneficial effect of pregnancy yoga interventions for anxiety, depression and perceived stress. Duration of labour was shorter (MD $=-116.96 ; 95 \% \mathrm{Cl}-163.36$ to $-70.56, \mathrm{P}<0.00001)$ and normal vaginal birth rates were higher in the yoga group (OR 2.72; 95\% $\mathrm{Cl} 1.26-5.90, p=0.01)$. The quality of evidence (GRADE) was low to very low for all factors. Twelve or more yoga sessions delivered weekly/bi-weekly had a statistically significant impact on mode of birth, while twelve or more yoga sessions of long duration (>60mins) had a statistically significant impact on perceived stress. Yoga sessions had a statistically significant impact on anxiety while yoga therapy had a statistically significant impact on depression

Conclusion: The evidence supports previously cited positive effects of pregnancy yoga on anxiety, depression, perceived stress, normal vaginal birth and shorter duration of labour.

Systematic review registration: PROSPERO, CRD42019119916. Registered on $11^{\text {th }}$ January 2019.

Protocol publication: Corrigan L, Eustace-Cook J, Moran P and Daly D. The effectiveness and characteristics of pregnancy yoga interventions: a systematic review protocol [version 2; peer review: 2 approved]. HRB Open Res 2020, 2:33 (https://doi.org/10.12688/hrbopenres.12967.2)

\section{Background}

Pregnancy is characterised by significant physiological, social and emotional changes which can impact on maternal and fetal health and wellbeing across multiple domains. ${ }^{(1,2)}$ There is comprehensive evidence that anxiety, depression, and stress in pregnancy are risk factors for adverse maternal and fetal outcomes ranging from preterm birth and low birth weight to adverse neurodevelopmental outcomes in infants and children. ${ }^{(3,4)}$ The well-being of the mother is therefore critical for optimal pregnancy and child outcomes. Pregnant women should be provided with support, tools, resources, and appropriate types and amounts of physical activity during pregnancy to reduce the risk of complications and promote optimal pregnancy and birth outcomes. ${ }^{(5)}$

Yoga is a mind-body-spirit practice combining physical postures, relaxation, and breathing techniques. ${ }^{(6,7)}$ It has been adapted for the pregnant body and is a common form of physical activity used by pregnant women and recommended by both healthcare and medical professionals. ${ }^{(7,8,9,10)}$ Evidence suggests that the practise of yoga in pregnancy is safe, feasible and acceptable to pregnant women and may be more beneficial than walking and standard prenatal exercises for both physical and mental health. $(5,11,12)$ It is also thought to provide pregnant women with the opportunity to foster well-being and develop a connection with their baby. ${ }^{(5,13)}$ RCTs of pregnancy yoga report that it lowers levels of pain, stress, anxiety and depression. $(14,15,16,17,18,19)$

A systematic review of yoga for pregnant women with six studies and 689 participants concluded that overall the evidence in support of pregnancy yoga was positive with randomised control trials (RCTs) indicating improvements in stress levels, quality of life (QoL), autonomic nervous system functioning and labour parameters such as comfort, pain and duration. ${ }^{(2)}$ A further systematic review identified wide variation in the length and intensity of the yoga interventions, the degree of supervision of the interventions, measurement tools utilised, sample population and outcomes measured, and recommended further exploration of these factors in future trials and analyses. ${ }^{(20)}$ Two recent meta-analyses one with 8 studies and one with 7 studies demonstrated that yoga was an effective complementary treatment to manage prenatal depression and improve mode of birth outcomes. ${ }^{(21,22)}$ Both studies identified limitations of women commencing yoga training at different gestational ages with varied frequency, type and intensity of yoga interventions across trials. While the body of evidence supporting the positive impact of yoga on pregnancy and birth outcomes is growing there is a need to pool evidence from studies to accurately measure treatment effect and explore the mechanisms by which yoga contributes to reported benefits. ${ }^{(2,20)}$ This should include analysis of the characteristics of the pregnancy yoga interventions in order to design programmes that can offer optimal benefit.

The objective of this systematic review was to examine the published evidence on pregnancy yoga, describe the characteristics of each intervention using the frequency, intensity, time/duration and type (FITT) principle of exercise prescription and through meta-analysis, assess the overall effects of pregnancy yoga on a range of identified outcomes. ${ }^{(23)}$ It will also critically review the methodological quality of the studies to guide future research. Understanding the factors that contribute to effective pregnancy yoga interventions can support the development of future pregnancy yoga programmes that optimise effect, ensure safety of mother and baby, and highlight how to best incorporate the practice into antenatal care. (20)

\section{Materials And Methods}




\section{Protocol}

This systematic review and meta-analysis were planned and conducted in accordance with Preferred reporting Items for Systematic Reviews and MetaAnalyses (PRISMA) guidelines (additional file 1), the PROSPERO registered (CRD42019119916) and HRBopen published protocols and the recommendations of the Cochrane Collaboration. $(24,25,26)$ The protocol was registered a priori.

\section{Search Strategy}

The following electronic databases were searched from their inception through May 2020:

(EBSCO)Medline (1946-), CINAHL (1981-), PsycINFO (1990-), (Ovid)Embase (1966-), AMED (1985-), WHOLiS, Web of Science (1864-), ScieLo (2002-) and ASSIA (1970-). The literature search was constructed around search terms for "pregnancy" and "yoga".

The search strategy was adapted for each database as necessary. Additional file 2 contains search terms and a search strategy for EMBASE. Reference lists from included studies and relevant reviews were screened to ensure all relevant studies were identified. No language or date of study publication restrictions were included in the search. The review only includes peer-reviewed published studies. The search was updated on 22nd May 2020.

\section{Selection Criteria}

\section{Participants}

Included studies involved both normal healthy and high-risk pregnant women of any gestation, age, ethnicity and country of residence.

\section{Intervention methods}

Studies where yoga was the primary intervention delivered to a sample of pregnant women. Multimodal interventions delivering yoga in conjunction with other treatments for pregnant women were excluded.

\section{Comparison methods}

Studies with pregnant women of any gestation receiving usual care or any active treatment other than yoga.

\section{Outcomes}

The primary outcomes of interest were stress, anxiety, depression and quality of life. Secondary outcomes were birth outcomes of labour duration, pain management in labour and mode of birth. Included studies had to assess at least one primary or secondary outcome measured using validated self-report or clinician-rated questionnaires, measures or scales or by clinical diagnosis or medical chart review.

\section{Study design}

Any primary study that investigated a pregnancy yoga intervention within a randomised control trial (RCT) or quasi-experimental study with a control before and after design was considered for inclusion. Case control studies, crossover trials and cross-sectional studies were excluded.

\section{Information retrieval and data extraction}

Literature search results were exported to EndNote X9 and duplicate records deleted using the 'remove duplicates' function and by manually screening results for accuracy (LC and JEC). ${ }^{(27)}$ Titles and abstracts of identified citations were imported to Covidence (JEC), a web-based software platform designed to support citation screening and collaboration among multiple authors. ${ }^{(28)}$

Two review authors (LC and DD) independently screened abstracts identified during the literature search and read potentially eligible articles in full to determine whether they met eligibility criteria. Disagreements were discussed with a third review author (PM) until consensus was reached. Two reviewers independently screened the full text of potentially eligible studies to determine inclusion/exclusion (LC and DD), with third-party arbitration (PM) available if needed. Reasons for excluding studies at full text review were recorded. The Preferred Reporting Items for Systematic Review and Meta-Analysis (PRISMA) flow diagram was used to show the overall process of study selection and summarise the inclusion and exclusion of studies at each stage of the review. (25)

A standardised data extraction tool (Additional file 3) was developed specifically for this review based on recommendations provided in the Cochrane Handbook of Systematic Reviews of Interventions (LC). ${ }^{(26)}$ Pairs of two authors (LC. and NMcG; LC and PM) independently extracted data on study design and methods, sociodemographic characteristics, inclusion and exclusion criteria, study setting, details of experimental intervention and comparison intervention, duration of follow-up and outcomes studied, and extent of effectiveness. Discrepancies were discussed with a fourth review author (DD) until consensus was reached. If necessary, study authors were contacted up to three times to provide further details. Data were entered into the RevMan5.3 software provided by the Cochrane collaboration network and checked for accuracy (LC). (29)

\section{Quality Assessment and assessment of confidence in the review findings}

The Cochrane collaboration's tool for assessing risk of bias provided by the Cochrane handbook for systematic reviews of intervention version 6.1 was used to evaluate the quality of the studies. ${ }^{(30)}$ Risk of bias assessment was undertaken by pairs of two authors (LC and NMcG; LC and PM) independently. Discrepancies were resolved by discussion with a fourth reviewer (DD), if required. Where reported information was unclear or where data were missing all attempts were made to contact the primary authors for clarification.

Quality of the evidence was evaluated using the Grades of Recommendation, Assessment, Development and Evaluation (GRADE) approach. (31) GRADEpro GDT software was used to import data from Review Manager Software to create the 'Summary of findings' table. ${ }^{(32,29)}$ Two review authors (LC and PM) graded the quality of the evidence for each outcome. Lack of double blinding alone was not downgraded due to difficulties of blinding participants and yoga 
instructors. Downgrading was based on risk of bias only if a lack of blinding was accompanied by additional high risks of bias (e.g. selection bias and incomplete outcome reporting). It should be noted that the GRADE approach this tool was developed for use in RCTs where double blinding was possible. (31)

A summary of intervention effects and a measure of quality according to the GRADE approach was determined for the following seven outcomes of interest:

- Maternal stress

- Maternal anxiety

- Maternal depression

- Maternal quality of life

- Duration of labour

- Pain management

- Mode of birth

Results from included studies are presented as summary risk ratios (RR) or odds ratios (OR) with $95 \%$ confidence intervals (Cl) for dichotomous outcomes. The mean difference (MD) was used for continuous data where outcomes were measured in the same way between trials. Where trials measured the same outcome using different methods the standardised mean difference (SMD) was used. The outcome measures from the individual trials were combined through meta-analysis where possible (clinical comparability of populations, interventions, outcomes and time of assessment between trials) using a randomeffects model, because we anticipated some between-study variation and according the Cochrane handbook for systematic reviews of intervention version 6.1 this offers the most conservative estimate. ${ }^{(30)}$ Data from studies that were too dissimilar to combine in a meta-analysis were described qualitatively in the text. Statistical heterogeneity was assessed in each meta-analysis using the $\mathrm{T}^{2}, \mathrm{I}^{2}$ and chi square statistics. ${ }^{(30)}$

Subgroup analysis applying the FITT principle of exercise prescription to stratify results by frequency, intensity, time/duration and type, where appropriate, was conducted. Any statistically significant subgroup effect was reported using the $\mathrm{p}$-value from the test for subgroup differences. The $\mathrm{I}^{2}$ statistic was used to measure the magnitude of heterogeneity in each sub-group and categorised according the Cochrane Handbook for Systematic Reviews of Interventions:

- "heterogeneity might not be important" (12 value $0 \%-40 \%)$

- "moderate heterogeneity" ( ${ }^{2}$ value $\left.30-60 \%\right)$

- "substantial heterogeneity" ( $1^{2}$ value $\left.50-90 \%\right)$

- "considerable heterogeneity" (I² value $75-100 \%){ }^{(30)}$

Sensitivity analysis to compare including and excluding RCTs at high risk of bias was conducted for stress (perceived), depression, duration of labour and mode of birth based on identification of studies with notably higher risk of bias.

\section{Results}

\section{Results of the search}

Figure 1 outlines the search and selection process. The total records identified through database searching and other sources were 679. Six hundred and twenty-four were excluded based on the title and abstract and 31 following full text review. Twenty-four studies including 2022 pregnant women were included in the review and the sample size ranged from 20-335. Data from 22 studies including 1826 pregnant women were suitable for and included in the metaanalysis.

\section{Study Characteristics}

Table 1 outlines the characteristics of the included studies and applies the frequency, intensity, time/duration and type (FITT) principle of exercise prescription across the studies. Ten of the included studies originated from India $(14,16,17,33-35,36-39)$, eight from the USA $(9,11,15,18,42,43-45)$ and one each from China, Indonesia, Iran, Japan, Thailand and the UK. ${ }^{(46-51)}$ Twenty of the studies were RCTs, three were non-randomised control trials and one was a trueexperimental post-test only control group design. Fifteen studies were conducted with normal healthy pregnant women $(9,14,17,35,36,38,39,44,46-51)$, two with multi-factor high risk pregnant women $(16,33)$, five with pregnant women with depression or symptoms of depression $(11,15,18,42,43)$, one with pregnant women with gestational diabetes ${ }^{(40)}$ and one with high risk pregnant women on bedrest. ${ }^{(45)}$ The gestational age at recruitment across studies ranged from $12-36$ weeks. All studies used a comparison group. Control groups were; routine antenatal care ( $n=12$ studies), usual activity ( $n=2$ studies), standard antenatal exercise ( $n=3$ studies), walking 30 minutes twice daily $(n=2$ studies), health education $(n=2$ studies) social support ( $n=1$ study), mom-baby wellness workshops ( $n=1$ study), and parenting education sessions ( $n=1$ study). 
Table 1

Characteristics of studies including FITT principle

\begin{tabular}{|c|c|c|c|c|c|c|c|c|c|}
\hline Study ID & Country & Study type & $\begin{array}{l}\text { Sample } \\
\text { size }\end{array}$ & $\begin{array}{l}\text { Gestation } \\
\text { (weeks) }\end{array}$ & Intervention & Control & $\begin{array}{l}\text { Outcome of } \\
\text { interest }\end{array}$ & Main results & Frequen \\
\hline $\begin{array}{l}\text { Babbar et al } \\
2016\end{array}$ & USA & $\begin{array}{l}\text { RCT } \\
\text { uncomplicated } \\
\text { pregnancy }\end{array}$ & $\begin{array}{l}46 \\
(23 / 23)\end{array}$ & $\begin{array}{l}28-36 \\
\text { weeks }\end{array}$ & $\begin{array}{l}\text { Yoga } \\
\text { session }\end{array}$ & $\begin{array}{l}\text { PowerPoint } \\
\text { presentation }\end{array}$ & Mode of birth & $\begin{array}{l}\text { NVB } 65 \% \text { yoga } \\
\text { and } 61 \% \\
\text { control }\end{array}$ & One timı \\
\hline $\begin{array}{l}\text { Balaji et al } \\
2017\end{array}$ & India & $\begin{array}{l}\text { RCT gestational } \\
\text { diabetes }\end{array}$ & $\begin{array}{l}151 \\
(75 / 76)\end{array}$ & 24 weeks & $\begin{array}{l}\text { Yoga } \\
\text { sessions }\end{array}$ & $\begin{array}{l}\text { Routine } \\
\text { treatment }\end{array}$ & Mode of birth & $\begin{array}{l}\text { NVB } 84 \% \text { yoga } \\
26 \% \text { control }\end{array}$ & Daily \\
\hline $\begin{array}{l}\text { Bershadsky } \\
\text { et al } 2014\end{array}$ & USA & $\begin{array}{l}\text { Non-randomised } \\
\text { control trial normal } \\
\text { pregnancy }\end{array}$ & $\begin{array}{l}50 \\
(38 / 12)\end{array}$ & $\begin{array}{l}12-19 \\
\text { weeks }\end{array}$ & $\begin{array}{l}\text { Yoga } \\
\text { sessions }\end{array}$ & Usual activity & $\begin{array}{l}\text { Depression } \\
\text { Physiological } \\
\text { stress }\end{array}$ & $\begin{array}{l}\text { Cortisol levels } \\
\text { lower post yoga } \\
\text { and fewer } \\
\text { depressive } \\
\text { symptoms in } \\
\text { yoga group }\end{array}$ & $\begin{array}{l}\text { No } \\
\text { informa }\end{array}$ \\
\hline $\begin{array}{l}\text { Bhartia et al } \\
2019\end{array}$ & India & $\begin{array}{l}\text { RCT low risk } \\
\text { pregnant women }\end{array}$ & $\begin{array}{l}78 \\
(38 / 40)\end{array}$ & $\begin{array}{l}18-20 \\
\text { weeks }\end{array}$ & $\begin{array}{l}\text { Yoga } \\
\text { therapy }\end{array}$ & $\begin{array}{l}\text { Routine } \\
\text { physical } \\
\text { activity }\end{array}$ & $\begin{array}{l}\text { Perceived } \\
\text { stress } \\
\text { Mode of birth }\end{array}$ & $\begin{array}{l}\text { Perceived } \\
\text { stress reduced } \\
31.75 \% \text { in yoga } \\
\text { group and } \\
\text { increased } \\
6.60 \% \text { in control } \\
(\mathrm{p}<0.001) \text {. NVB } \\
92 \% \text { yoga and } \\
90 \% \text { control }\end{array}$ & Tri-week \\
\hline $\begin{array}{l}\text { Bolanthakodi } \\
\text { et al } 2018\end{array}$ & India & $\begin{array}{l}\text { RCT normal } \\
\text { pregnancy }\end{array}$ & $\begin{array}{l}150 \\
(75 / 75)\end{array}$ & 30 weeks & $\begin{array}{l}\text { Yoga } \\
\text { therapy }\end{array}$ & $\begin{array}{l}\text { Standard } \\
\text { antenatal } \\
\text { care }\end{array}$ & $\begin{array}{l}\text { Mode of birth } \\
\text { Pain } \\
\text { management }\end{array}$ & $\begin{array}{l}\text { More NVB in } \\
\text { yoga group ( } p< \\
0.037) \text {, duration } \\
\text { of labour was } \\
\text { significantly } \\
\text { shorter } \\
(p<0.001) \\
\text { Significant } \\
\text { reduction in } \\
\text { intravenous } \\
\text { analgesic } \\
\text { in yoga group } \\
\text { ( } p<0.045) \text { and } \\
\text { tolerance of } \\
\text { pain was higher } \\
\text { as shown by } \\
\text { NPIS ( } p<0.001 \text { ) } \\
\text { and PBOS } \\
\text { scores } \\
\text { ( } p<0.0001)\end{array}$ & $\begin{array}{l}\text { Bi-weekl } \\
\text { for } 4 \\
\text { sessions } \\
\text { and we } € \\
\text { for three } \\
\text { sessions } \\
\text { and self } \\
\text { practice } \\
\text { weekly }\end{array}$ \\
\hline $\begin{array}{l}\text { Chen et al } \\
2017\end{array}$ & China & $\begin{array}{l}\text { RCT healthy } \\
\text { pregnant women }\end{array}$ & $\begin{array}{l}94 \\
(48 / 46)\end{array}$ & 16 weeks & $\begin{array}{l}\text { Yoga } \\
\text { sessions }\end{array}$ & $\begin{array}{l}\text { Routine } \\
\text { prenatal care }\end{array}$ & $\begin{array}{l}\text { Physiological } \\
\text { stress }\end{array}$ & $\begin{array}{l}\text { Prenatal yoga } \\
\text { significantly } \\
\text { reduced cortisol } \\
(p<0.001)\end{array}$ & Bi-weekl \\
\hline $\begin{array}{l}\text { Chuntharapat } \\
\text { et al } 2008\end{array}$ & Thailand & $\begin{array}{l}\text { RCT normal } \\
\text { pregnancy }\end{array}$ & $\begin{array}{l}74 \\
(37 / 37)\end{array}$ & $\begin{array}{l}26-28 \\
\text { weeks }\end{array}$ & $\begin{array}{l}\text { Yoga } \\
\text { sessions }\end{array}$ & $\begin{array}{l}\text { Routine } \\
\text { nursing care }\end{array}$ & $\begin{array}{l}\text { Pain } \\
\text { management } \\
\text { Duration of } \\
\text { labour }\end{array}$ & $\begin{array}{l}\text { No differences } \\
\text { between groups } \\
\text { for pethidine } \\
\text { usage. Shorter } \\
\text { duration of } \\
\text { labour in yoga } \\
\text { group }\end{array}$ & $\begin{array}{l}\text { Bi-weekl } \\
\text { taught a } \\
\text { tri-weekl } \\
\text { self- } \\
\text { practice }\end{array}$ \\
\hline $\begin{array}{l}\text { Davis et al } \\
2015\end{array}$ & USA & $\begin{array}{l}\text { RCT symptoms } \\
\text { anxiety/depression }\end{array}$ & $\begin{array}{l}46 \\
(23 / 23)\end{array}$ & 28 weeks & $\begin{array}{l}\text { Yoga } \\
\text { sessions }\end{array}$ & TAU & $\begin{array}{l}\text { Depression } \\
\text { Anxiety }\end{array}$ & $\begin{array}{l}\text { Prenatal yoga } \\
\text { was associated } \\
\\
\text { with reductions } \\
\text { in symptoms of } \\
\text { anxiety and } \\
\text { depression }\end{array}$ & Weekly \\
\hline
\end{tabular}




\begin{tabular}{|c|c|c|c|c|c|c|c|c|c|}
\hline Study ID & Country & Study type & $\begin{array}{l}\text { Sample } \\
\text { size }\end{array}$ & $\begin{array}{l}\text { Gestation } \\
\text { (weeks) }\end{array}$ & Intervention & Control & $\begin{array}{l}\text { Outcome of } \\
\text { interest }\end{array}$ & Main results & Frequen \\
\hline $\begin{array}{l}\text { Deshpande et } \\
\text { al } 2013\end{array}$ & India & $\begin{array}{l}\text { RCT high risk } \\
\text { pregnancies }\end{array}$ & $\begin{array}{l}68 \\
(30 / 38)\end{array}$ & 12 weeks & $\begin{array}{l}\text { Yoga } \\
\text { therapy }\end{array}$ & $\begin{array}{l}\text { Standard } \\
\text { antenatal } \\
\text { care/prenatal } \\
\text { stretching } \\
\text { exercises }\end{array}$ & $\begin{array}{l}\text { Perceived } \\
\text { stress }\end{array}$ & $\begin{array}{l}\text { RMANOVA } \\
\text { showed a } \\
\text { significant } \\
\text { decrease }(P= \\
0.02) \text { in the } \\
\text { PSS scores of } \\
\text { the yoga group } \\
\text { compared to } \\
\text { the control } \\
\text { group }\end{array}$ & $\begin{array}{l}\text { No } \\
\text { informa: }\end{array}$ \\
\hline $\begin{array}{l}\text { Field et al } \\
2012\end{array}$ & USA & RCT depression & $\begin{array}{l}56 \\
(28 / 28)\end{array}$ & 20 weeks & $\begin{array}{l}\text { Yoga } \\
\text { postures }\end{array}$ & $\begin{array}{l}\text { Standard } \\
\text { prenatal care }\end{array}$ & $\begin{array}{l}\text { Depression } \\
\text { Anxiety }\end{array}$ & $\begin{array}{l}\text { Decreased } \\
\text { depression } \\
\text { scores }(\mathrm{F}= \\
82.40, \\
\mathrm{p}<0.001) \text { and } \\
\text { decreased } \\
\text { anxiety scores } \\
(\mathrm{F}=26.23, \mathrm{p}< \\
0.001) \text { in the } \\
\text { yoga group }\end{array}$ & Bi-weekl \\
\hline $\begin{array}{l}\text { Field et al } \\
2013\end{array}$ & USA & RCT depression & $\begin{array}{l}92 \\
(46 / 46)\end{array}$ & 22 weeks & $\begin{array}{l}\text { Yoga } \\
\text { postures }\end{array}$ & $\begin{array}{l}\text { Social } \\
\text { support }\end{array}$ & $\begin{array}{l}\text { Depression } \\
\text { Anxiety } \\
\text { Physiological } \\
\text { stress }\end{array}$ & $\begin{array}{l}\text { Reduced } \\
\text { anxiety and } \\
\text { depression in } \\
\text { both groups } \\
\text { with no } \\
\text { significant } \\
\text { group } \\
\text { difference and } \\
\text { reduced cortisol } \\
\text { pre/post yoga } \\
\text { and pre/post } \\
\text { social support }\end{array}$ & Weekly \\
\hline $\begin{array}{l}\text { Gallagher et } \\
\text { al } 2020\end{array}$ & USA & $\begin{array}{l}\text { RCT high risk } \\
\text { pregnancy on } \\
\text { bedrest }\end{array}$ & $\begin{array}{l}79 \\
(48 / 31)\end{array}$ & $\begin{array}{l}23-31 \\
\text { weeks }\end{array}$ & $\begin{array}{l}\text { Yoga } \\
\text { sessions }\end{array}$ & $\begin{array}{l}\text { Standard } \\
\text { care and no } \\
\text { yoga }\end{array}$ & $\begin{array}{l}\text { Depression } \\
\text { Anxiety }\end{array}$ & $\begin{array}{l}\text { Perceived } \\
\text { anxiety and } \\
\text { depression } \\
\text { overall scores } \\
\text { lower in yoga } \\
\text { group than in } \\
\text { control group } \\
(p<0.001)\end{array}$ & $\begin{array}{l}\text { Bi-weekl } \\
\text { taught, } \\
\text { video se } \\
\text { practice }\end{array}$ \\
\hline $\begin{array}{l}\text { Hayase et al } \\
2018\end{array}$ & Japan & $\begin{array}{l}\text { Non-randomised } \\
\text { control trial } \\
\text { uncomplicated } \\
\text { pregnancy }\end{array}$ & $\begin{array}{l}91 \\
(38 / 53)\end{array}$ & $\begin{array}{l}20-23 \\
\text { weeks }\end{array}$ & $\begin{array}{l}\text { Yoga } \\
\text { sessions }\end{array}$ & $\begin{array}{l}\text { Standard } \\
\text { antenatal } \\
\text { care }\end{array}$ & $\begin{array}{l}\text { Perceived } \\
\text { stress } \\
\text { Physiological } \\
\text { stress }\end{array}$ & $\begin{array}{l}\text { PSS scores } \\
\text { lower in yoga } \\
\text { group at } 20-23 \\
\text { \& } 28-31 \text { weeks' } \\
\text { gestation. } \\
\text { Salivary a- } \\
\text { amylase levels } \\
\text { in yoga group } \\
\text { significantly } \\
\text { decreased } \\
\text { immediately } \\
\text { after yoga }\end{array}$ & $\begin{array}{l}\text { Weekly } \\
\text { taught a } \\
\text { daily sel } \\
\text { practice }\end{array}$ \\
\hline
\end{tabular}




\begin{tabular}{|c|c|c|c|c|c|c|c|c|c|}
\hline Study ID & Country & Study type & $\begin{array}{l}\text { Sample } \\
\text { size }\end{array}$ & $\begin{array}{l}\text { Gestation } \\
\text { (weeks) }\end{array}$ & Intervention & Control & $\begin{array}{l}\text { Outcome of } \\
\text { interest }\end{array}$ & Main results & Frequen \\
\hline $\begin{array}{l}\text { Jahdi et al } \\
2017\end{array}$ & Iran & $\begin{array}{l}\text { RCT normal } \\
\text { pregnancy }\end{array}$ & $\begin{array}{l}60 \\
(30 / 30)\end{array}$ & 26 weeks & $\begin{array}{l}\text { Yoga } \\
\text { sessions }\end{array}$ & $\begin{array}{l}\text { Routine } \\
\text { midwifery } \\
\text { care }\end{array}$ & $\begin{array}{l}\text { Mode of birth } \\
\text { Duration of } \\
\text { labour } \\
\text { Pain } \\
\text { management }\end{array}$ & $\begin{array}{l}\text { Duration of the } \\
\text { second and } \\
\text { third stages of } \\
\text { labour } \\
\text { significantly } \\
\text { shorter in yoga } \\
\text { group ( } p=0.04 \\
\text { and } 0.01 \\
\text { respectively). } \\
\text { Caesarean } \\
\text { section rate } \\
13.3 \% \text { in yoga } \\
\text { group } \\
\text { compared to } \\
50 \% \text { in control } \\
\text { group. } \\
\text { Analgesic use } \\
\text { during first } \\
\text { stage of labour } \\
\text { showed no } \\
\text { difference } \\
\text { between groups } \\
\text { ( }=0.2 \text { ) }\end{array}$ & $\begin{array}{l}\text { Tri-week } \\
\text { taught } 8 \\
\text { daily sel } \\
\text { practice }\end{array}$ \\
\hline $\begin{array}{l}\text { Mitchell et al } \\
2012\end{array}$ & USA & RCT depression & $\begin{array}{l}24 \\
(12 / 12)\end{array}$ & 20 weeks & $\begin{array}{l}\text { Yoga } \\
\text { postures }\end{array}$ & $\begin{array}{l}\text { parenting } \\
\text { education } \\
\text { sessions }\end{array}$ & Depression & $\begin{array}{l}\text { Depressive } \\
\text { symptoms } \\
\text { reduced to } \\
\text { subclinical } \\
\text { levels in } 55 \% \text { of } \\
\text { yoga group } \\
\text { compared to } \\
11 \% \text { of control } \\
\text { group }\end{array}$ & Bi-weekl \\
\hline $\begin{array}{l}\text { Munirekha et } \\
\text { al } 2019\end{array}$ & India & $\begin{array}{l}\text { True-experimental } \\
\text { post-test } \\
\text { only control group } \\
\text { design - } \\
\text { uncomplicated } \\
\text { pregnancy }\end{array}$ & $\begin{array}{l}30 \\
(15 / 15)\end{array}$ & $\begin{array}{l}24-32 \\
\text { weeks }\end{array}$ & $\begin{array}{l}\text { Yoga } \\
\text { sessions }\end{array}$ & $\begin{array}{l}\text { Health } \\
\text { education on } \\
\text { antenatal } \\
\text { care and } \\
\text { future } \\
\text { lactation }\end{array}$ & Mode of birth & $\begin{array}{l}\text { NVB } 80 \% \text { yoga } \\
\text { group } \\
\text { compared to } \\
40 \% \text { control } \\
\text { group }\end{array}$ & Weekly \\
\hline $\begin{array}{l}\text { Narendran et } \\
\text { al } 2005\end{array}$ & India & $\begin{array}{l}\text { Non-randomised } \\
\text { control trial normal } \\
\text { pregnancy }\end{array}$ & $\begin{array}{l}335 \\
(169 / 166)\end{array}$ & $\begin{array}{l}18-20 \\
\text { weeks }\end{array}$ & $\begin{array}{l}\text { Yoga } \\
\text { therapy }\end{array}$ & $\begin{array}{l}\text { Walking } 30 \\
\text { mins twice } \\
\text { daily }\end{array}$ & Mode of birth & $\begin{array}{l}\text { NVB 54\% yoga } \\
\text { group } \\
\text { compared to } \\
49 \% \text { control } \\
\text { group }\end{array}$ & Daily \\
\hline $\begin{array}{l}\text { Newham et al } \\
2014\end{array}$ & UK & $\begin{array}{l}\text { RCT healthy } \\
\text { pregnant women }\end{array}$ & $\begin{array}{l}59 \\
(31 / 28)\end{array}$ & $\begin{array}{l}20-24 \\
\text { weeks }\end{array}$ & $\begin{array}{l}\text { Yoga } \\
\text { sessions }\end{array}$ & TAU & $\begin{array}{l}\text { Anxiety } \\
\text { Depression } \\
\text { Physiological } \\
\text { stress }\end{array}$ & $\begin{array}{l}\text { Greater } \\
\text { reduction in } \\
\text { both anxiety } \\
\text { and depression } \\
\text { in the yoga } \\
\text { group. } \\
\text { Significant } \\
\text { decrease in } \\
\text { cortisol after } \\
\text { yoga }(0.15 \\
{[0.11] \mu \mathrm{g} / \mathrm{dL} \mathrm{vs} \text {. }} \\
0.13[0.10] \mathrm{\mu g} / \mathrm{dL} \\
\mathrm{P}=0.003)\end{array}$ & Weekly \\
\hline
\end{tabular}




\begin{tabular}{|c|c|c|c|c|c|c|c|c|c|}
\hline Study ID & Country & Study type & $\begin{array}{l}\text { Sample } \\
\text { size }\end{array}$ & $\begin{array}{l}\text { Gestation } \\
\text { (weeks) }\end{array}$ & Intervention & Control & $\begin{array}{l}\text { Outcome of } \\
\text { interest }\end{array}$ & Main results & Frequen \\
\hline $\begin{array}{l}\text { Rakhshani et } \\
\text { al } 2010\end{array}$ & India & $\begin{array}{l}\text { RCT normal } \\
\text { pregnancy }\end{array}$ & $\begin{array}{l}102 \\
(51 / 51)\end{array}$ & $\begin{array}{l}18-20 \\
\text { weeks }\end{array}$ & $\begin{array}{l}\text { Integrated } \\
\text { yoga }\end{array}$ & $\begin{array}{l}\text { Standard } \\
\text { antenatal } \\
\text { exercises }\end{array}$ & Quality of life & $\begin{array}{l}\text { Between } \\
\text { groups analysis } \\
\text { showed } \\
\text { significant } \\
\text { improvements } \\
\text { in the yoga } \\
\text { group in the } \\
\text { physical } \\
(P=0.001) \text {, } \\
\text { psychological } \\
(P=0.001) \text {, } \\
\text { social }(P= \\
0.003) \\
\text { environmental } \\
\text { domains }(P= \\
0.001) \text { of the } \\
\text { WHOQOL-100 }\end{array}$ & Tri-week \\
\hline $\begin{array}{l}\text { Rakhshani et } \\
\text { al } 2012\end{array}$ & India & $\begin{array}{l}\text { RCT high risk } \\
\text { pregnancy }\end{array}$ & $\begin{array}{l}68 \\
(30 / 38)\end{array}$ & 12 weeks & $\begin{array}{l}\text { Integrated } \\
\text { yoga }\end{array}$ & $\begin{array}{l}\text { Standard } \\
\text { care plus } \\
\text { walking for } \\
\text { half an hour } \\
\text { mornings } \\
\text { and evenings }\end{array}$ & Mode of birth & $\begin{array}{l}\text { Lower rate of } \\
\text { emergency c- } \\
\text { section in yoga } \\
\text { group } 51.7 \% \\
\text { compared to } \\
57.9 \% \text { in control }\end{array}$ & Tri-week \\
\hline $\begin{array}{l}\text { Satyapriya et } \\
\text { al } 2009\end{array}$ & India & $\begin{array}{l}\text { RCT normal } \\
\text { pregnancy }\end{array}$ & $\begin{array}{l}90 \\
(45 / 45)\end{array}$ & $\begin{array}{l}18-20 \\
\text { weeks }\end{array}$ & $\begin{array}{l}\text { Integrated } \\
\text { yoga }\end{array}$ & $\begin{array}{l}\text { Standard } \\
\text { prenatal } \\
\text { exercise }\end{array}$ & $\begin{array}{l}\text { Perceived } \\
\text { stress }\end{array}$ & $\begin{array}{l}\text { Perceived } \\
\text { stress } \\
\text { decreased by } \\
31.57 \% \text { in the } \\
\text { yoga group and } \\
\text { increased by } \\
6.60 \% \text { in the } \\
\text { control group } \\
(P=0.001)\end{array}$ & $\begin{array}{l}\text { Tri-week } \\
\text { for first } \\
\text { month tl } \\
\text { daily sel } \\
\text { practice }\end{array}$ \\
\hline $\begin{array}{l}\text { Satyapriya et } \\
\text { al } 2013\end{array}$ & India & $\begin{array}{l}\text { RCT normal } \\
\text { pregnancy }\end{array}$ & $\begin{array}{l}96 \\
(51 / 45)\end{array}$ & $\begin{array}{l}18-20 \\
\text { weeks }\end{array}$ & $\begin{array}{l}\text { Integrated } \\
\text { yoga }\end{array}$ & $\begin{array}{l}\text { Standard } \\
\text { antenatal } \\
\text { exercises }\end{array}$ & $\begin{array}{l}\text { Anxiety } \\
\text { Depression } \\
\text { Perceived } \\
\text { stress }\end{array}$ & $\begin{array}{l}\text { Anxiety and } \\
\text { Depression } \\
\text { reduced with } \\
\text { improvement in } \\
\text { pregnancy } \\
\text { experience in } \\
\text { the yoga group } \\
(\mathrm{P}<0.001)\end{array}$ & $\begin{array}{l}\text { Tri-week } \\
\text { for first } \\
\text { month tl } \\
\text { daily sel } \\
\text { practice }\end{array}$ \\
\hline $\begin{array}{l}\text { Uebelacker et } \\
\text { al } 2016\end{array}$ & USA & RCT depression & $20(12 / 8)$ & $\begin{array}{l}12-26 \\
\text { weeks }\end{array}$ & $\begin{array}{l}\text { Yoga } \\
\text { sessions }\end{array}$ & $\begin{array}{l}\text { Mom-baby } \\
\text { wellness } \\
\text { workshops }\end{array}$ & Depression & $\begin{array}{l}\text { Although both } \\
\text { groups had } \\
\text { reduced } \\
\text { depression } \\
\text { scores, yoga } \\
\text { was preferred. }\end{array}$ & Weekly \\
\hline $\begin{array}{l}\text { Yulianti et al } \\
2018\end{array}$ & Indonesia & $\begin{array}{l}\text { RCT normal } \\
\text { pregnancy }\end{array}$ & $\begin{array}{l}102 \\
(51 / 51)\end{array}$ & $\begin{array}{l}22-32 \\
\text { weeks }\end{array}$ & $\begin{array}{l}\text { Yoga } \\
\text { sessions }\end{array}$ & Not treated & $\begin{array}{l}\text { Depression } \\
\text { Anxiety }\end{array}$ & $\begin{array}{l}\text { Mean level of } \\
\text { anxiety and } \\
\text { depression } \\
\text { were lower in } \\
\text { the yoga group } \\
\text { at both two and } \\
\text { four weeks post } \\
\text { intervention (p } \\
<0.001 \text { ) }\end{array}$ & $\begin{array}{l}\text { No } \\
\text { informa: }\end{array}$ \\
\hline
\end{tabular}

\section{Characteristics of pregnancy yoga interventions}

The frequency of the pregnancy yoga intervention ranged from once off to daily, the session length ranged from 20-120 minutes and the intensity ranged from a single session to availability of 126 practice sessions. Four studies classified the yoga intervention as yoga therapy ${ }^{(16,35,36,39)}$, thirteen yoga sessions $(9,11,37,38,43-51)$, three yoga postures $(15,18,42)$ and four integrated yoga. $(14,17,33,34)$ All yoga interventions used physical postures. Of the 24 included studies, 21 did not define the specific style of yoga used in the intervention; two stated that hatha yoga was used and one stated that Ashtanga Vinyasa was used. ${ }^{(43,}$ 
44,51) The interventions included ten studies with taught sessions only $(9,15,18,33,34,38,42,44,46,51)$, twelve with taught sessions plus self-practice using manuals or DVDs $(14,17,35-37,39,41,43,45,48-50)$ and two studies did not provide adequate information on the mode of delivery of the yoga intervention. ${ }^{(16,47)}$ Risk of bias

Overall, most studies were assessed as having a high risk of bias for at least one domain with no studies rating low risk of bias across all domains. Figure 2 shows the overall risk of bias assessment across domains and the risk of bias in each included study.

Allocation While 14 RCTs used adequate methods of random sequence generation, only eight adequately concealed allocation.

Blinding Only eight studies blinded outcome assessors. Twenty-three studies explicitly reported to not have blinded participants to treatment allocation; the remaining study did not report on blinding of participants and personnel. Given the difficulties of blinding behavioural interventions, participants and personnel were most likely not blinded to the allocated yoga intervention in this trial.

Incomplete outcomes data Five studies rated high risk of attrition bias due to evidence of incomplete data, inclusion of completers only in the analysis and subjects moving groups not being reported.

Selective reporting All 24 studies were considered low risk for reporting bias.

Other potential sources of bias Eleven studies were rated high risk of other bias due to exclusion of participants from the final analysis without explanation, baseline imbalances, loss to follow-up imbalances across groups, self-selection bias, self-reports of compliance lack of clarity on the administration of the yoga intervention and use of insensitive instruments to measure outcomes. The proportion of information from studies at high risk of bias was sufficient to affect the interpretation of results.

\section{Assessment of the quality of the evidence - GRADE}

The quality assessment for individual review outcomes informed by the GRADEpro Guideline Development Tool (GDT) are reported in Table 2 summary of findings for yoga versus treatment as usual or any other active treatment. There was low quality evidence that pregnancy yoga interventions could be effective for each outcome included in this review, perceived stress, physiological stress, anxiety, depression, duration of labour, mode of birth and quality of life. 


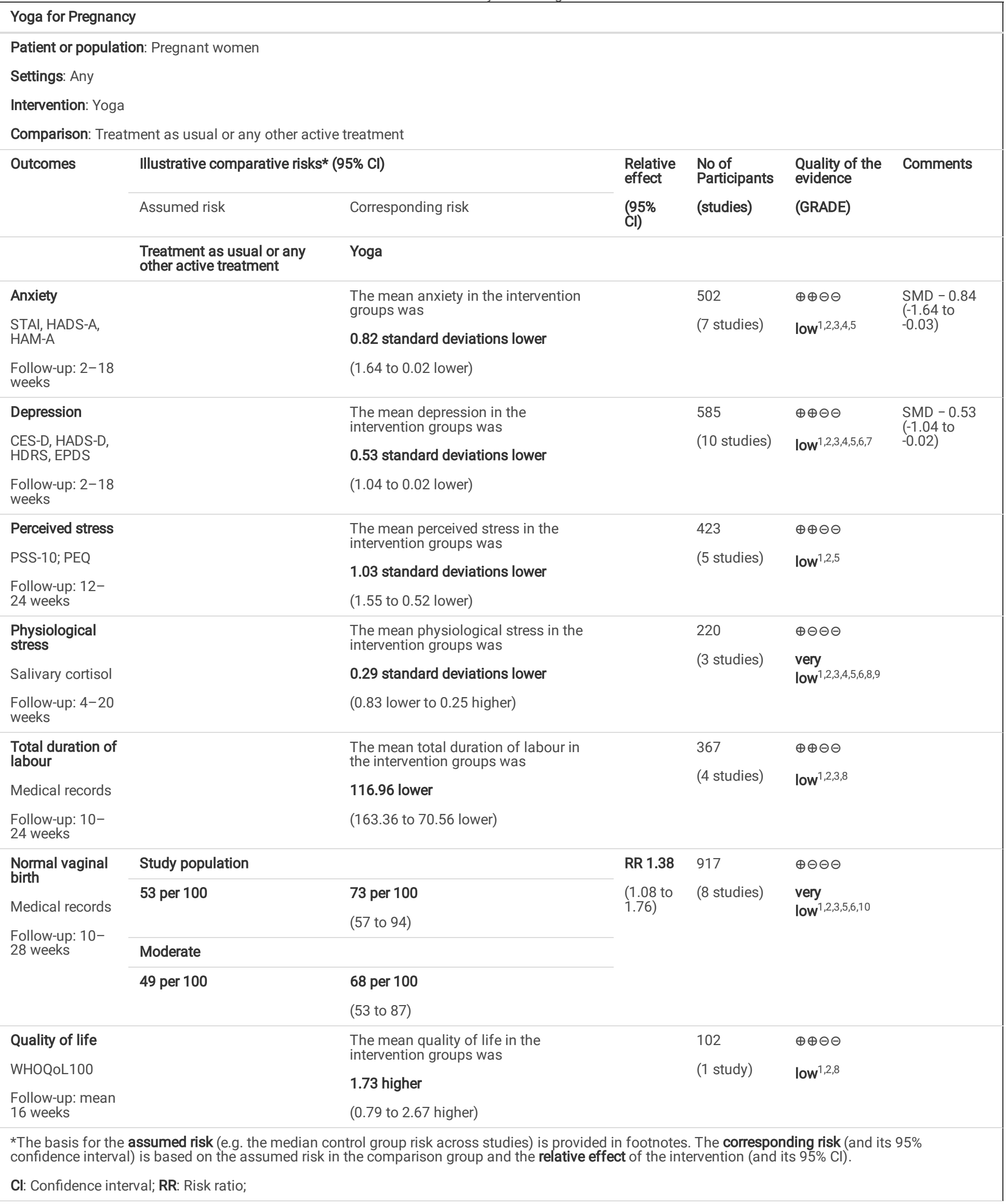




\section{Yoga for Pregnancy}

GRADE Working Group grades of evidence

High quality: Further research is very unlikely to change our confidence in the estimate of effect.

Moderate quality: Further research is likely to have an important impact on our confidence in the estimate of effect and may change the estimate.

Low quality: Further research is very likely to have an important impact on our confidence in the estimate of effect and is likely to change the estimate.

Very low quality: We are very uncertain about the estimate.

${ }^{1}$ Concerns with high risk of bias for allocation concealment

${ }^{2}$ Concerns with high risk of bias for lack of blinding of participants

${ }^{3}$ Concerns with high risk of bias due to unclear evidence on blinding of outcome assessors

${ }^{4}$ Serious inconsistency due to large variation in effect across studies

${ }^{5}$ Serious inconsistency 12 value is large indicating substantial heterogeneity

${ }^{6}$ Concerns with high risk of bias for random allocation

${ }^{7}$ Concerns with high risk of bias due to pre-existing depression or depressive symptoms in some studies

${ }^{8}$ Serious imprecision based on total population size $>400$

${ }^{9}$ Serious imprecision due to wide $95 \% \mathrm{Cls}$

${ }^{10}$ Serious inconsistency due to inclusion of high risk pregnant populations

Abbreviations: STAI - state and trait anxiety scale, HADS-A - hospital anxiety and depression scale - anxiety, HAM-A - Hamilton anxiety rating scale, CESD - centre for epidemiological studies - depression, HADS-D - hospital anxiety and depression scale - depression, HDRS - Hamilton depression rating scale, EPDS - Edinburgh postnatal depression scale, PSS-10 - perceived stress scale 10 item, PEQ - pregnancy experiences questionnaire.

\section{Primary Outcomes}

\section{Stress}

Five RCTs with 423 participants reported post intervention perceived stress scores measured by the Perceived Stress Scale (PSS-10) in four studies and the Pregnancy Experiences Questionnaire (PEQ) in one study. $(14,16,17,39,49)$ The pooled SMD $(-1.03 ; 95 \%$ Cl: -1.55 to $-0.52 ; p<0.0001)$ supports a statistically significant beneficial effect of pregnancy yoga interventions for perceived stress. (Fig. 3a) A sensitivity analysis removing a study at high risk of bias supported these results and lowered heterogeneity ( $\left.\operatorname{Tau}^{2}=0.14, \mathrm{I}^{2}=70 \% ; \mathrm{p}<0.00001\right)$. ${ }^{(49)}$ (Fig. 3b) Three RCTs with 220 participants reported post intervention stress levels, measured by salivary cortisol. $(18,44,49)$ The pooled SMD $(-0.29 ; 95 \% \mathrm{Cl}:-0.83$ to $0.25 ; p=0.30)$ demonstrated no significant effect for physiological stress. (Fig. 3c) A further two RCTs reported data on physiological stress but were not suitable for meta-analysis. ${ }^{(46,51)}$ Chen et al. looked at short-term and long-term stress and immunological effects of yoga in 94 healthy pregnant women. ${ }^{(46)}$ Salivary cortisol levels were obtained immediately before and after intervention at 16,20,24, 28, 32, and 36 weeks gestation for both groups. Baseline characteristics and pre-test salivary cortisol levels were similar between both groups. Although yoga displayed a short-term decrease in cortisol, there were no significant differences in long-term cortisol effects between groups. The second RCT conducted by Newham et al with 29 pregnant women reported that salivary cortisol levels were significantly lower immediately after the yoga intervention. ${ }^{(51)}$

\section{Anxiety}

Seven RCTs with 502 participants reported post intervention anxiety symptom scores measured by the state-trait anxiety scale (STAl), hospital anxiety and depression scale - anxiety (HADS-A) and hamilton anxiety rating scale (HAM-A). ${ }^{(15,17,18,43,45,47,51)}$ The pooled SMD (-0.82; $95 \%$ Cl: -1.64 to $\left.-0.01 ; p=0.05\right)$ supports a statistically significant beneficial effect of pregnancy yoga interventions for anxiety. (Fig. 3d)

\section{Depression}

Ten RCTs with 585 participants reported post intervention depression symptom scores measured by centre for epidemiological studies - depression (CES-D), hospital anxiety and depression scale - depression (HADS-D), Edinburgh postnatal depression scale (EPDS) and hamilton depression rating scale (HDRS). (11, $15,17,18,42-45,47,51)$ The pooled SMD $(-0.53 ; 95 \%$ Cl: -1.04 to $-0.02 ; p=0.04)$ supports a statistically significant beneficial effect of pregnancy yoga interventions for depression symptoms. (Fig. 3e) Sensitivity analysis performed to remove a study with high risk of bias from the analysis showed no difference. ${ }^{(47)}$

\section{Quality of life}

One RCT with 102 participants reported post intervention quality of life scores measured by the world health organisation quality of life assessment instrument (WHOQoL-100). ${ }^{(34)}$ Between group analysis showed significant improvements in the yoga group compared to the control in the physical (15.79 \pm 2.77 (15-16.570, $p=0.001)$, psychological (16.08 $\pm 2.12(15-16.57), p<0.001)$, social relationships $(16.88 \pm 1.91(16.34-17.42), P=0.003)$ and 
environmental domains (16.25 $\pm 2(15.69-16.82), P=0.001)$. Results were not significant for independence $(15.91 \pm 2.2(15.29-16.53), P=0.065)$ and spiritual domains $(16.02 \pm 2.42(15.34-16.70), P=0.23)$.

\section{Secondary outcomes}

\section{Labour duration}

Four RCTs with 367 participants reported data on the duration of labour. $(36,48-50)$ The pooled MD calculated in minutes (-116.96; $95 \%$ Cl: -163.36 to -70.56 ; $p$ $<0.00001)$ supports a statistically significant beneficial effect of pregnancy yoga interventions for shorter duration of labour by an average of almost two hours. (Fig. 4a) Sensitivity analysis performed to remove a study with high risk of bias from the analysis showed no difference. (49)

\section{Pain management}

Three RCTs with 276 participants reported data on pain management during labour. ${ }^{(36,48,50)}$ Data from these studies were not suitable for meta-analysis. One study reported on single or multiple doses of tramadol reporting significant reduction in requirement of intravenous analgesic in the pregnancy yoga group ( $\mathrm{p}$ $<0.045)$. Tolerance of pain was also increased in the pregnancy yoga group as measured by the numerical pain intensity scale (NPIS) ( $<<0.001)$ and pain behavioural observation scale (PBOS) $(p<0.0001) .{ }^{(36)}$ A second study found that the pregnancy yoga group demonstrated significantly higher maternal comfort during labour as measured by the visual analogue sensation of pain scale (VASPS) and PBOS $(p<0.05)$ while no differences were found, between the groups, regarding pethidine usage. ${ }^{(50)}$ A further study reported that analgesic use during the first stage of labour showed no difference between groups $(p=$ 0.2). ${ }^{(48)}$

\section{Mode of birth}

Eight studies with 917 participants reported data on the mode of birth. $(9,14,33,35-39)$ Compared to control groups the vaginal birth rate was significantly higher in the pregnancy yoga groups (OR $=2.72 ; 95 \% \mathrm{Cl}: 1.26-5.90 ; \mathrm{p}<0.00001)$. (Fig. $4 \mathrm{~b})$ Sensitivity analysis performed to remove three studies with a focus on high risk pregnancies, with an implied increased risk of a caesarean birth, from the analysis maintained an increased likelihood of a vaginal birth in the pregnancy yoga group $(\mathrm{OR}=1.81 ; 95 \% \mathrm{Cl}: 1.00-3.27 ; \mathrm{p}=0.05) .{ }^{(33,37,38)}$ (Fig. $\left.4 \mathrm{c}\right)$ As expected, removing these studies also reduced heterogeneity $\left(\right.$ Tau ${ }^{2}=0.20$, $\mathrm{I}^{2}=46 \% ; \mathrm{P}=0.05$ compared to $\left.\mathrm{Tau}^{2}=0.93, \mathrm{I}^{2}=81 \% ; \mathrm{p}=0.01\right)$.

\section{Subgroup FITT principle analysis Frequency}

Six of the 24 included studies (25\%) reported that pregnant women were assigned to a moderate frequency (three times weekly) yoga intervention ( $14,17,33,34$, $39,48)$, twelve studies (50\%) assigned pregnant women to a low frequency (once or twice weekly) yoga intervention $(11,15,18,36,38,42,43,45,46,49-51)$ and two studies (8\%) assigned pregnant women to a high frequency (daily) yoga intervention. ${ }^{(35,37)}$ Three studies $(13 \%)$ did not report the frequency of the pregnancy yoga intervention $(16,44,47)$ and one study $(4 \%)$ offered just a single session. ${ }^{(40)}$

Subgroup analysis of the frequency of the yoga intervention across outcomes could not be conducted for perceived stress, anxiety, depression, duration of labour, pain management or quality of life. This was due to the small numbers of studies in each subgroup making comparisons impractical. The test for subgroup differences for mode of birth suggest that there is a statistically significant subgroup effect for low frequency yoga interventions of weekly or biweekly sessions $(p=0.006)$. (Fig. $5 a)$ However, a smaller number of trials and participants contributed data to the subgroup, meaning that the analysis may not be able to accurately detect subgroup differences. There is low heterogeneity between the trials within the subgroup $\left(I^{2}=0 \%\right)$ suggesting the validity of the treatment effect estimate is reliable.

\section{Intensity}

Intensity was assessed based on taught yoga sessions as opposed to self-reported home practice. One study (4\%) had just a single yoga session ${ }^{(9)}$, nine

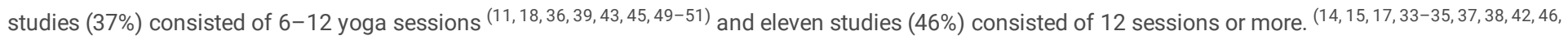
48) Three studies (13\%) did not provide adequate information for analysis of intensity. $(16,44,47)$

Subgroup analysis looking at the intensity of the yoga interventions across outcomes was not possible for duration of labour, pain management or quality of life. The test for subgroup differences for perceived stress revealed that there is statistically significant subgroup effect for interventions with more than 12 sessions $(p<0.00001)$. (Fig. $5 b)$ There is low heterogeneity between the trials within this subgroup $\left(I^{2}=0 \%\right)$ meaning the validity of the treatment effect estimate is reliable. The small number of trials and participants, however, means that the analysis is not powered to accurately detect subgroup differences. The analysis for anxiety revealed that $6-12$ sessions had a more significant impact on anxiety $(p=0.02)$ than more than 12 sessions. (Fig. $5 c)$ There was moderate heterogeneity between the trials within this subgroup $\left(I^{2}=48 \%\right)$, therefore the validity of the treatment effect estimate is uncertain. Again, the small number of trials and participants mean the subgroup differences lack reliability. For depression, the analysis demonstrated no statistically significant difference for interventions with $6-12$ sessions $(p=0.09)$ or more than 12 sessions $(p=0.24)$. (Fig. $5 d)$ An analysis of mode of birth showed that interventions with more than 12 sessions had a more significant positive impact on normal vaginal births $(p=0.03)$. (Fig. $5 e)$ There was considerable heterogeneity between the trials within this subgroup $\left(I^{2}=89 \%\right)$ meaning the validity of the treatment effect estimate is unreliable.

\section{Timing}


Five studies (21\%) used short-time duration ( $\leqq 45 \mathrm{~min}$ ) yoga sessions $(15,18,36,42,45)$, nine studies $(37 \%)$ used moderate-time duration $(>45-\leqq 60$ min) yoga sessions $^{(9,33-35,37,39,48-50)}$ and seven studies (29\%) used a long duration session time (>60min). ${ }^{(11,14,17,43,44,46,51)}$ Three studies $(13 \%)$ did not report the duration of the yoga sessions. $(16,38,47)$

Subgroup analysis looking at the timing of the yoga intervention across outcomes could not be carried out for duration of labour, mode of birth, pain management and quality of life. The test for subgroup differences for perceived stress demonstrated that long duration yoga interventions greater than 60 minutes had a greater significant positive impact $(p<0.00001)$ than moderate duration yoga interventions greater than 45 minutes but less than 60 minutes. (Fig. 5f) There was low heterogeneity between the trials within this subgroup $\left(1^{2}=0 \%\right)$ meaning the validity of the treatment effect estimate is reliable. The small number of trials and participants, however, mean that the analysis is not powered to accurately detect subgroup differences. The same subgroup analysis for anxiety revealed no significant difference between short $\leqq 45$ minutes $(p=0.59)$ ) and long duration $>60$ minutes yoga interventions on anxiety ( $p$ $=0.09$ ). (Fig. $5 g$ ). For depression, subgroup analysis demonstrated no significant difference in impact on depression scores between short $(p=0.16)$ and long duration yoga interventions $(p=0.27)$. (Fig. 5h)

\section{Type}

Thirteen studies (54\%) reported the intervention consisted of a yoga session $(9,11,37,38$, 43-51), eight studies (33\%) yoga therapy $(14,16,17,33-35,36,39)$ and three studies $(13 \%)$ yoga postures. $(15,18,42)$ In addition, two studies $(8 \%)(44,51)$ named the type of yoga as Hatha and one study $(4 \%)$ as Ashtanga Vinyasa. $(43)$

Subgroup analysis of the type of the yoga intervention provided across outcomes was not feasible for perceived stress, duration of labour, pain management and quality of life. The test for subgroup differences for anxiety suggest that there is a statistically significant subgroup effect for yoga sessions ( $p=0.02)$ and yoga therapy $(p<0.00001)$ compared to yoga postures $(p=0.55)$. (Fig. $5 i)$ However, a smaller number of trials and participants contributed data to the therapy subgroup than to the postures and sessions subgroups, meaning that the analysis may not be able to accurately detect subgroup differences. There is moderate heterogeneity between the trials within each of these subgroups (postures: $I^{2}=44 \%$; sessions: $I^{2}=48 \%$ ), therefore the validity of the treatment effect estimate for each subgroup is uncertain. The analysis for depression indicates a statistically significant subgroup effect for yoga therapy ( $p<0.00001)$ but not for yoga postures $(p=0.55)$ and yoga sessions $(p=0.25)$. (Fig. $5 j)$ However, there was only one trial in the therapy subgroup, meaning the analysis could not accurately detect subgroup differences. The test for subgroup difference for mode of birth revealed that there is a statistically significant subgroup effect for yoga sessions $(p=0.005)$ but not for yoga therapy $(p=0.10)$. (Fig. $5 k)$ There is substantial heterogeneity between the trials within the yoga sessions subgroup $\left(I^{2}=74 \%\right)$ meaning the validity of the treatment effect estimate is unreliable.

\section{Discussion}

The aim of this systematic review was to examine the published evidence on pregnancy yoga, describe the characteristics of each intervention using the frequency, intensity, time/duration and type (FITT) principle of exercise prescription and through meta-analysis, assess the overall effects of pregnancy yoga on stress, anxiety, depression, quality of life, labour duration, pain management in labour and mode of birth. Twenty-four trials with a total of 2022 pregnant women met the inclusion criteria and 22 trials with 1826 pregnant women were suitable for inclusion in the meta-analysis.

The first question this review addressed was: what are the characteristics of pregnancy yoga interventions using the FITT principle? Data extracted from the 24 studies showed that the frequency of the pregnancy yoga interventions ranged from once off to daily, session length varied from $20-120$ minutes and intensity fluctuated from a single session to availability of 126 practice sessions. Only 3 studies specifically named a type of yoga with two citing the use of Hatha yoga and one Ashtanga Vinyasa. ${ }^{(43,44,51)}$ Regarding the content of the yoga intervention $2^{(16,47)}$ of the studies failed to provide details, $3{ }^{(15,18,42)}$ described solely physical postures and the remaining 19 a practice that included postures, breathwork, meditation and relaxation. (14, 17,33-41, 43-51) The studies were not consistent in frequency, intensity, duration or type of yoga intervention and the content of the interventions varied widely. Of note 10 studies included taught sessions only $(9,15,18,33,34,38,42,44,46,51), 12$ taught sessions plus self-practice using manuals or DVDs (11, 14, 17, 35-37, 39, 43, 45, 48-50) and 2 studies did not provide adequate information on the method of delivery of the yoga intervention. ${ }^{(16,47)}$ According to Mottola \& Artal, 2016 in order to provide safe exercise guidelines, pregnant women should be prescribed exercises in accordance with the FITT principle. ${ }^{(52)}$ Future studies should focus on specifying the frequency, intensity, duration and type of yoga in order to better understand the components of the intervention that impact optimally on both pregnancy outcomes and safety. This could then facilitate the development of a checklist of essential components for an evidence-based pregnancy yoga practice that could be replicated.

The second question this review examined was: what are the effects of pregnancy yoga interventions on stress, anxiety, depression, quality of life, labour duration, pain management in labour and mode of birth? The results of the meta-analysis suggest that yoga is a beneficial

non-pharmacological intervention to manage levels of stress, anxiety and depression. Applying per-protocol analysis and a random effects model, the pooled standardised mean difference (SMD) for depression ( $-0.53 ; 95 \% \mathrm{Cl}:-1.04$ to $-0.02, \mathrm{P}=0.04)$, anxiety $(-0.82 ; 95 \% \mathrm{Cl}:-1.64$ to $-0.01 ; \mathrm{p}=0.05)$, perceived stress $(-1.03 ; 95 \% \mathrm{Cl}:-1.55$ to $-0.52 ; \mathrm{p}<0.0001)$ and physiological stress $(-0.24 ; 95 \% \mathrm{Cl}$ : -0.52 to $0.04, \mathrm{P}=0.09)$ supported a statistically significant beneficial effect of pregnancy yoga interventions for anxiety, depression and perceived stress. The variable methods of both measuring and reporting stress, anxiety and depression across studies may have contributed to the elevated degree of heterogeneity seen in the analysis. It is also acknowledged that many of the tools used were not diagnostic tools but measures of symptomatology with study participants exhibiting normal range or mildly elevated scores at baseline rather than moderate-severe levels of stress, anxiety or depression. One RCT with 102 participants reported post intervention quality of life scores and between group analysis using the WHOQoL-100 showed significant improvements in the yoga group compared to the control in the physical, psychological, social relationships and environmental domains. ${ }^{(34)}$ The participants in this RCT were experiencing normal, healthy pregnancies. Future studies targeting high-risk pregnant women are suggested to see if similar results could be obtained. In relation to birth outcomes this meta-analysis showed that duration of labour was

Page $13 / 22$ 
shorter by on average up to 2 hours and women in the yoga group were 2.7 times more likely to experience a normal vaginal birth. These findings are supported by a previous qualitative review that examined yoga and its efficacy with 10 of the 15 studies demonstrating positive changes in maternal psychological or birth outcome measures. ${ }^{(53)}$ A recent meta-analysis also found that yoga was an effective complementary and alternative therapy in promoting vaginal births and shortening the first and second stages of labour. ${ }^{(22)}$ Three RCTs with 276 participants reported data on pain management during labour. ${ }^{(36,48,50)}$ Data from these studies were not suitable for meta-analysis. One study reported significant reduction in requirement of intravenous analgesic in the pregnancy yoga group. ${ }^{(36)}$ A second study found that the pregnancy yoga group demonstrated significantly higher maternal comfort during labour and no differences were found, between the groups, regarding pethidine usage. ${ }^{(50)}$ A third study reported that analgesic use during the first stage of labour showed no difference between groups. Previous research on yoga for pain management suggests that clinically meaningful changes in pain can be observed through the use of yoga for a multitude of conditions. ${ }^{(54-57)}$ There is however a paucity of research in the area and further understanding of the mechanisms by which yoga can influence and modify the pain response are needed.

The third question this review examined was: what are the FITT principles that contribute optimally to effectiveness outcomes on stress, anxiety, depression, quality of life, labour duration, pain management in labour and mode of birth? In this study low frequency yoga interventions of weekly or bi-weekly sessions had a more significant impact on mode of birth. This finding is encouraging as during pregnancy women can be burdened with multiple appointments, work and family commitments which would make attending more than bi-weekly sessions a challenge. In relation to intensity there was a statistically significant subgroup effect for interventions with more than 12 sessions for perceived stress and mode of birth and interventions of 6-12 sessions for anxiety. This is the first meta-analysis to suggest the optimal number of sessions to maximise effect and future trials can use this data to plan sessions numbers based on their intended outcomes. Regarding duration subgroup differences for perceived stress demonstrated that long duration yoga interventions greater than 60 minutes had a greater significant positive impact. This can support planning of session duration for future trials and indeed pregnancy yoga classes in the community. As only 3 studies specifically named a type of yoga the interventions were split into yoga sessions, yoga therapy and yoga postures based on the components of the interventions described in the studies. The results of meta-analysis showed that for anxiety there was a statistically significant subgroup effect for both yoga sessions and yoga therapy. The analysis for depression indicates a statistically significant subgroup effect for yoga therapy and for mode of birth there is a statistically significant subgroup effect for yoga sessions. These findings can support the incorporation of the FITT principle into the design of interventions for future trials based on the target population and the outcome measures of interest.

Of note we found no evidence of adverse events in any of the trials, suggesting that yoga is a safe practice during pregnancy. In terms of limitations results highlight issues regarding lack of allocation concealment and double-blinding, attrition bias, small sample sizes, a wide variety of outcome measures, nonstandardised or replicable yoga interventions, lack of measurement of fidelity to the intervention and huge variation in the components of the yoga interventions. Many studies used self-practice which is difficult to monitor for both compliance and safety. High levels of compliance and safety are important for interventions to be effective so future studies should consider how the intervention is delivered and monitored. This will improve fidelity and potentially maximise effect. Of the 24 included studies 10 originated from India $(14,16,17,33-35,36-39)$, 8 from the USA $(9,15,18,41-45)$ and one each from China, Indonesia, Iran, Japan, Thailand and the UK. ${ }^{(46-51)}$ A recent systematic review demonstrated that RCTs on yoga that are conducted in India have are about 25 times more likely to reach positive conclusions than those conducted elsewhere. ${ }^{58)}$ Further in-depth studies are recommended to elucidate reasons for the differences in conclusions between Indian RCTs and those conducted elsewhere, and it may be beneficial to report on Indian trials separately in future reviews. Finally, women in the included studies were of middle-to-high socioeconomic status, presenting a selection bias of participants thus reducing generalisability. Further studies should be conducted on at-risk populations from lower socio-economic backgrounds.

The results of this study offer positive insights into the characteristics and effectiveness of pregnancy yoga and support its potential role in improving maternal and birth outcomes. However, the quality of the evidence (GRADE) was low to very low for all factors. Therefore, more high-quality studies are needed before the efficacy of pregnancy yoga interventions for maternal and birth outcomes can be definitively known. Future studies of yoga for pregnancy should ensure rigorous trial design and reporting and evidence-informed intervention development.

\section{Conclusion}

The present review and meta-analysis offer valuable information on the characteristics and effectiveness of pregnancy yoga interventions. The evidence supports previously cited positive effects of pregnancy yoga on anxiety, depression, perceived stress, normal vaginal birth and shorter duration of labour. Recommendations above can be used to support researchers to work collaboratively with yoga practitioners to standardise pregnancy yoga interventions and conduct more robust evidence-based evaluation. Overall, the evidence supporting yoga in pregnancy is growing, but methodological weaknesses with published studies and an insufficient number of published RCTs with reproducible evidence-based interventions highlight the need for further research.

\section{Abbreviations}

ADHD - Attention deficit hyperactivity disorder

CES-D - Centre for epidemiological studies - depression

$\mathrm{Cl}$ - Confidence interval

EPDS - Edinburgh postnatal depression scale

FITT - frequency, intensity, time/duration and type

GRADE - Grades of recommendation, assessment, development and evaluation 
HADS-A - Hospital anxiety and depression scale - anxiety

HADS-D - Hospital anxiety and depression scale - depression

HAM-A - Hamilton anxiety rating scale

HDRS - Hamilton depression rating scale

$\mathrm{OR}$ - Odds ratio

$\mathrm{PIH}$ - Pregnancy induced hypertension

PRISMA-P - Preferred reporting items for systematic reviews and meta-analysis protocols

PSS-10 - Perceived stress scale 10 item

QoL - Quality of life

RCT - Randomised control trial

RR - Risk ratio

STAI - The state-trait anxiety inventory

WHOQoL-100 - World health organisation quality of life assessment instrument

\section{Declarations}

\section{Acknowledgements}

The authors acknowledge the support of both the Health Research Board, Ireland and the SPHeRE programme, Ireland in providing scholarship funding to the corresponding author to complete her doctoral studies.

\section{Funding}

Health Research Board, Ireland (SPHeRE/2013/1). The funders had no role in study design, data collection and analysis, decision to publish, or preparation of the manuscript.

\section{Authors' Contributions}

LC, PM, JEC and DD conceptualised the review, LC and JEC designed the search strategy and performed the searches, LC, PM, NMcG and DD performed the data extraction, risk of bias and GRADE assessments, LC performed the meta-analysis and wrote the first draft of the manuscript, PM, NMcG, JEC and DD provided critical feedback and edits to the draft. All authors approved the final manuscript.

\section{Ethics approval and consent to participate}

Ethical approval was not required for this study as it did not involve conducting experimental research, nor include identifying personal data. The systematic review is being disseminated in peer-reviewed journals.

\section{Consent for publication}

Not applicable

\section{Availability of data and material}

The dataset generated and/or analysed during the study are available from the corresponding author on reasonable request.

\section{Competing interests}

The authors declare that they have no competing interests.

\section{Author details}

${ }^{1}$ School of Nursing and Midwifery, Trinity College Dublin, Dublin 2, Ireland

2 The Library of Trinity College Dublin, Dublin 2, Ireland.

${ }^{3}$ School of Public Health, University College Cork, Cork, Ireland.

\section{References}


1. Littleton HL, Bye K, Buck K, et al.: Psychosocial stress during pregnancy and perinatal outcomes: a meta-analytic review. J Psychosom Obstet Gynaecol. 2010; 31(4): 219-228. DOI: 3109/0167482X.2010.518776. Epub 2010 Nov 2.

2. Curtis K, Weinrib A, Katz J: Systematic review of yoga for pregnant women: current status and future directions. Evid Based Complement and Alternat Med. 2012; 2012: 715942. DOI: 1155/2012/715942. Epub 2012 Aug 14.

3. Dunkel Schetter C, Tanner L. Anxiety, depression and stress in pregnancy: implications for mothers, children, research, and practice. Curr Opin Psychiatry. 2012; 25(2): 141-148. DOI:1097/YCO.0b013e3283503680

4. Baibazarova E, van de Beek C, Cohen-Kettenis PT, et al.: Influence of prenatal maternal stress, maternal plasma cortisol and cortisol in the amniotic fluid on birth outcomes and child temperament at 3 months. Psychoneuroendocrinology. 2013; 38(6): 907-915. DOI: 1016/j.psyneuen.2012.09.015.Epub 2012 Oct 7.

5. Jiang Q, Wu Z, Zhou L, Dunlop J, Chen P. Effects of yoga intervention during pregnancy: a review for current status. Am J Perinatol. 2015 May; $32(6)$ : 50314. DOI: 1055/s-0034-1396701. Epub 2014 Dec 23.

6. lyengar BKS. Light on Yoga. New York: Schocken; 1979. ISBN 0-8052-1031-8.

7. Curtis K, Weinrib A, Katz J. Systematic review of yoga for pregnant women: current status and future directions. Evid Based Complement and Alternat Med. 2012; 2012: 715942. DOI:1155/2012/715942.

8. Hall HR, Jolly K. Women's use of complementary and alternative medicines during pregnancy: a cross sectional study. Midwifery. 2014 May; 30(5): 499505. DOI: 1016/j.midw.2013.06.001.

9. Babbar 2016 Babbar S, Hill JB, Williams KB, Pinon M, Chauhan SP, Maulik D. Acute feTal behavioral Response to prenatal Yoga: a single, blinded, randomized controlled trial (TRY yoga). Am J Obstet Gynecol. 2016 Mar; 214(3): 399.e1-8. DOI: 1016/j.ajog.2015.12.032. Epub 2015 Dec 22.

10. Mooventhan A. A Comprehensive review on scientific evidence-based effects (including adverse effects) of yoga for normal and high-risk pregnancyrelated health problems. J Bodyw Mov Ther. 2019 ;23(4): DOI: https://doi.org/10.1016/j.jbmt.2019.03.005

11. Uebelacker LA, Battle CL, Sutton KA, Magee SR, Miller IW. A pilot randomized controlled trial comparing prenatal yoga to perinatal health education for antenatal depression. Arch Womens Ment Health. 2016 Jun; 19(3): 543-7. DOI: 1007/s00737-015-0571-7. Epub 2015 Sep 18.

12. Battle CL, Uebelacker LA, Magee SR, Sutton KA, Miller IW. Potential for prenatal yoga to serve as an intervention to treat depression during pregnancy. Womens Health Issues. 2015 Mar-Apr; 25(2): 134-41. DOI: 1016/j.whi.2014.12.003.

13. Kwon R, Kasper K, London S, Haas DM. A systematic review: the effects of yoga on pregnancy. Eur J Obstet Gynecol Reprod Biol. 2020 Jul; $250: 171-177$. DOI: https://doi.org/10.1016/j.ejogrb.2020.03.044

14. SatyapriyaM, Nagendra HR, Nagarathna R, Padmalatha V. Effect of integrated yoga on stress and heart rate variability in pregnant women. Int J Gynaecol Obstet 2009 Mar; 104(3): 218-222. DOI: 1016/j.ijgo.2008.11.013.Epub 2008 Dec 25.

15. Field T, Diego M, Hernandez-Reif M, Medina L, Delgado J, Hernandez A. Yoga and massage therapy reduce prenatal depression and prematurity. J Bodyw Mov Ther 2012 Apr; 16(2): 204-209. DOI: 1016/j.jbmt.2011.08.002. Epub 2011 Sep 23.

16. Deshpande C, Rakshani A, Nagarathna R, et al. Yoga for high-risk pregnancy: a randomized controlled trial. Ann Med Health Sci Res 2013 Jul-Sep; 3(3): 341-344. DOI:4103/2141-9248.117933.

17. Satyapriya M, Nagarathna R, Padmalatha V, Nagendra HR. Effect of integrated yoga on anxiety, depression \& wellbeing in normal pregnancy. Complement Ther Clin Pract. 2013 Nov; 19(4): 230-236. DOI: 1016/j.ctcp.2013.06.003.Epub 2013 Jul 26.

18. Field T, Diego M, Delgado J, Medina L. Yoga and social support reduce prenatal depression, anxiety and cortisol. J Bodyw Mov Ther. 2013 Oct; 17(4): 397-403. DOI: 1016/j.jpmt.2013.03.010.Epub 2013 May 3.

19. Martins RF, Pinto e Silva JL. Treatment of pregnancy-related lumbar and pelvic girdle pain by the yoga method: a randomized controlled study. J Altern Complement Med 2014 Jan; 20(1): 24-31. DOI: 1089/acm.2012.0715.Epub 2013 Mar 18.

20. Riley K, Drake E: The Effects of Prenatal Yoga on Birth Outcomes: A Systematic Review of the Literature. J Prenat Perinat Psychol Health. 2013; 28(1): 319.

21. Ng QX, Venkatanarayanan N, Loke W, Yeo WS, Lim DY, Chan HW et al. A meta-analysis of the effectiveness of yoga-based interventions for maternal depression during pregnancy. Complement Ther Clin Pract. 2019 Feb; 34: 8-12. DOI: 1016/j.ctcp.2018.10.016. Epub 2018 Oct 28.

22. Rong L, Dai LJ, Ouyang YQ. The effectiveness of prenatal yoga on delivery outcomes: A meta-analysis. Complement Ther Clin Pract. 2020 May; 39 : 101157. DOI: 1016/j.ctcp.2020.101157.Epub 2020 Mar 28.

23. American College of Sports Medicine in Riebe D, Ehrman JK, Liguori G, et al.: ACSM's guidelines for exercise testing and prescription. Tenth Edition. Philadelphia: Wolters Kluwer, 2018.

24. Corrigan L, Eustace-Cook J, Moran P and Daly D. The effectiveness and characteristics of pregnancy yoga interventions: a systematic review protocol [version 2; peer review: 2 approved]. HRB Open Res.2020; 2(33). DOI: https://doi.org/10.12688/hrbopenres.12967.2.

25. Moher D, Liberati A, Tetzlaff J, Altman DG PRISMA Group. Preferred reporting items for systematic reviews and meta-analyses: the PRISMA statement. J Clin Epidemiol. 2009 Oct; 62(10): 1006-12. DOI: 1016/j.jclinepi.2009.06.005.

26. Higgins JPT, Thomas J, Chandler J, Cumpston M, Li T, Page MJ, Welch VA, editors. Cochrane Handbook for Systematic Reviews of Interventions. 2nd Edition. Chichester (UK): John Wiley \& Sons, 2019.

27. Endnote $X 9$ software, Clarivate analystics.

28. Covidence systematic review software, Veritas health innovation, Melbourne, Australia. Available at www.covidence.org

29. RevMan. Review Manager. 53 $3^{\text {rd }}$ ed. Copenhagen: The Nordic Cochrane Centre, The Cochrane Collaboration; 2014.

Page $16 / 22$ 
30. Higgins JPT, Thomas J, Chandler J, Cumpston M, Li T, Page MJ, Welch VA (editors). Cochrane Handbook for Systematic Reviews of Interventionsversion 6.1 (updated September 2020). Cochrane, 2020. Available from training.cochrane.org/handbook.

31. Schünemann H, Brożek J, Guyatt G, Oxman A, editors. GRADE handbook for grading quality of evidence and strength of recommendations. Updated October 2013. The GRADE Working Group, 2013. Available from: guidelinedevelopment.org/handbook.

32. GRADEpro GDT: GRADEpro Guideline Development Tool [Software]. McMaster University, 2015 (developed by Evidence Prime, Inc.). Available from gradepro.org.

33. Rakhshani A, Nagarathna R, Mhaskar R, Mhaskar A, Thomas A, Gunasheela S. The effects of yoga in prevention of pregnancy complications in high-risk pregnancies: a randomized controlled trial. Prev Med. 2012 Oct; 55(4): 333-340. DOI: 1016/j.y.ymed.2012.07.020. Epub 2012 Aug 2. PMID: 22884667.

34. Rakhshani A, Maharana S, Raghuram N, Nagendra HR, Venkatram P. Effects of integrated yoga on quality of life and interpersonal relationship of pregnant women. Qual Life Res. 2010 Dec; 19(10): 1447-55. DOI: 1007/s11136-010-9709-2. Epub 2010 Jul 15. PMID: 20632115.

35. Narendran S, Nagarathna R, Narendran V, Gunasheela S, Nagendra HR. Efficacy of yoga on pregnancy outcome. J Altern Complement Med. 2005 Apr; 11(2): 237-44. DOI: 1089/acm.2005.11.237. PMID: 15865489.

36. Bolanthakodi C, Raghunandan C, Saili A, Mondal S, Saxena P. Prenatal Yoga: Effects on Alleviation of Labor Pain and Birth Outcomes. J Altern Complement Med. 2018 Dec; 24(12): 1181-1188. DOI: 1089/acm.2018.0079. Epub 2018 Aug 30. PMID: 30160530.

37. Balaji A, Varne S. Physiological effects of yoga asanas and pranayama on metabolic parameters, maternal, and fetal outcome in gestational diabetes. Natl J Physiol Pharm Pharmacol. 2017 Mar; 7(7): 724-728. DOI: 5455/njppp.2017.7.0306713032017

38. Munirekha B, Jayashree K, Vijayaraghavan R. A study to assess the effectiveness of antenatal exercises and yoga to promote normal vaginal deliveries among antenatal mothers. Int. J. Res. Ayurveda Pharm. 2019; 10(6): 85-90 DOI: http://dx.doi.org/10.7897/2277-4343.1006130

39. Bhartia N, Jain S, Shankar N, Rajaram S, Gupta M. Effects of Antenatal Yoga on Maternal Stress and Clinical Outcomes in North Indian Women: A Randomised Controlled Trial. J Indian Acad Clin Med. 2019 Jan-Mar; 20(1): 10-14.

40. Babbar S, Hill JB, Williams KB, Pinon M, Chauhan SP, Maulik D. Acute feTal behavioral Response to prenatal Yoga: a single, blinded, randomized controlled trial (TRY yoga). Am J Obstet Gynecol. 2016 Mar; 214(3): 399.e1-8. DOI: 1016/j.ajog.2015.12.032. Epub 2015 Dec 22. PMID: 26721782.

41. Uebelacker LA, Battle CL, Sutton KA, Magee SR, Miller IW. A pilot randomized controlled trial comparing prenatal yoga to perinatal health education for antenatal depression. Arch Womens Ment Health. 2016 Jun; 19(3): 543-7. DOI: 1007/s00737-015-0571-7. Epub 2015 Sep 18. PMID: 26385456; PMCID: PMC4798904.

42. Mitchell, J, Field, T, Diego M, Bendell D, Newton R, Pelaez M. Yoga Reduces Prenatal Depression Symptoms. Psychology. 2012 Sep; 3(9A), $782-786$. DOI: 4236/psych.2012.329118.

43. Davis K, Goodman SH, Leiferman J, Taylor M, Dimidjian S. A randomized controlled trial of yoga for pregnant women with symptoms of depression and anxiety. Complement Ther Clin Pract. 2015 Aug; 21(3): 166-72. DOI: 1016/j.ctcp.2015.06.005. Epub 2015 Jun 9. PMID: 26256135.

44. Bershadsky S, Trumpfheller L, Kimble HB, Pipaloff D, Yim IS. The effect of prenatal Hatha yoga on affect, cortisol and depressive symptoms. Complement Ther Clin Pract. 2014 May; 20(2): 106-13. DOI: 1016/j.ctcp.2014.01.002. Epub 2014 Jan 24. PMID: 24767955; PMCID: PMC4003457.

45. Gallagher A, Kring D, Whitley T. Effects of yoga on anxiety and depression for high risk mothers on hospital bedrest. Complement Ther Clin Pract. 2020 Feb; 38:101079. DOI: 1016/j.ctcp.2019.101079. Epub 2019 Dec 14. PMID: 32056815.

46. Chen PJ, Yang L, Chou CC, Li CC, Chang YC, Liaw JJ. Effects of prenatal yoga on women's stress and immune function across pregnancy: A randomized controlled trial. Complement Ther Med. 2017 Apr; 31: 109-117. DOI: 1016/j.ctim.2017.03.003. Epub 2017 Mar 7. PMID: 28434463.

47. Yulianti I, Respati SH, Sudiyanto The Effect of Prenatal Yoga on Anxiety and Depression in Kudus, Central Java. JMCH. 2018); 3(2): 100-104 DOI: https://doi.org/10.26911/thejmch.2018.03.02.02

48. Jahdi F, Sheikhan F, Haghani H, Sharifi B, Ghaseminejad A, Khodarahmian M, Rouhana N. Yoga during pregnancy: The effects on labor pain and delivery outcomes (A randomized controlled trial). Complement Ther Clin Pract. 2017 May; 27: 1-4. DOI: 1016/j.ctcp.2016.12.002. Epub 2016 Dec 23. PMID: 28438273.

49. Hayase M, Shimada M. Effects of maternity yoga on the autonomic nervous system during pregnancy. J Obstet Gynaecol Res. 2018 Oct; 44(10):1 8871895. DOI: 1111/jog.13729. Epub 2018 Jul 17. PMID: 30019537.

50. Chuntharapat S, Petpichetchian W, Hatthakit U. Yoga during pregnancy: effects on maternal comfort, labor pain and birth outcomes. Complement Ther Clin Pract. 2008 May; 14(2): 105-15. DOI: 1016/j.ctcp.2007.12.007. Epub 2008 Mar 4. PMID: 18396254.

51. Newham JJ, Wittkowski A, Hurley J, Aplin JD, Westwood M. Effects of antenatal yoga on maternal anxiety and depression: a randomized controlled trial. Depress Anxiety. 2014 Aug; 31(8): 631-40. DOI: 1002/da.22268. Epub 2014 Apr 30. PMID: 24788589.

52. Mottola MF, Artal R. Fetal and maternal metabolic responses to exercise during pregnancy. Early Human Development. 2016 ; 94 : 33-41.

53. Sharma M, Branscum P. Yoga interventions in pregnancy: a qualitative review. JACM. 2015 Apr; 21(4): 208-216. https://doi.org/10.1089/acm.2014.0033

54. Russell N, Daniels B, Smoot B, Allen, DD. Effects of yoga on quality of life and pain in women with chronic pelvic pain: systematic review and metaanalysis. JWHPT. 2019 Jul/Sep; 43(3): 144-154). DOI: 10.1097/JWH.0000000000000135

55. Lauche R, Hunter DJ, Adams J, Cramer H.Yoga for osteoarthritis: a systematic review and meta-analysis. Curr Rheumatol Rep. 2019: 21(47). https://doi.org/10.1007/s11926-019-0846-5.

56. Cramer H, Klose P, Brinkhaus B, Michalsen A, Dobos G. Effects of yoga on chronic neck pain: a systematic review and meta-analysis. Clinical Rehabilitation. 2017;31(11):1457-1465. DOI:1177/0269215517698735

57. Bussing A, Ostermann T, Ludtke R, Michalsen A. Effects of yoga interventions on pain and pain-associated disability: A meta-analysis. J Pain. 2012; 13: 1-9.

Page $17 / 22$ 
58. Cramer H, Lauche R, Langhorst J, Dobos G. Are Indian yoga trials more likely to be positive than those from other countries? A systematic review of randomized controlled trials. Contemp Clin Trials. 2015 Mar; 41: 269-272. DOI: 10.1016/j.cct.2015.02.005. Epub 2015 Feb 20. PMID: 25705015.

\section{Figures}

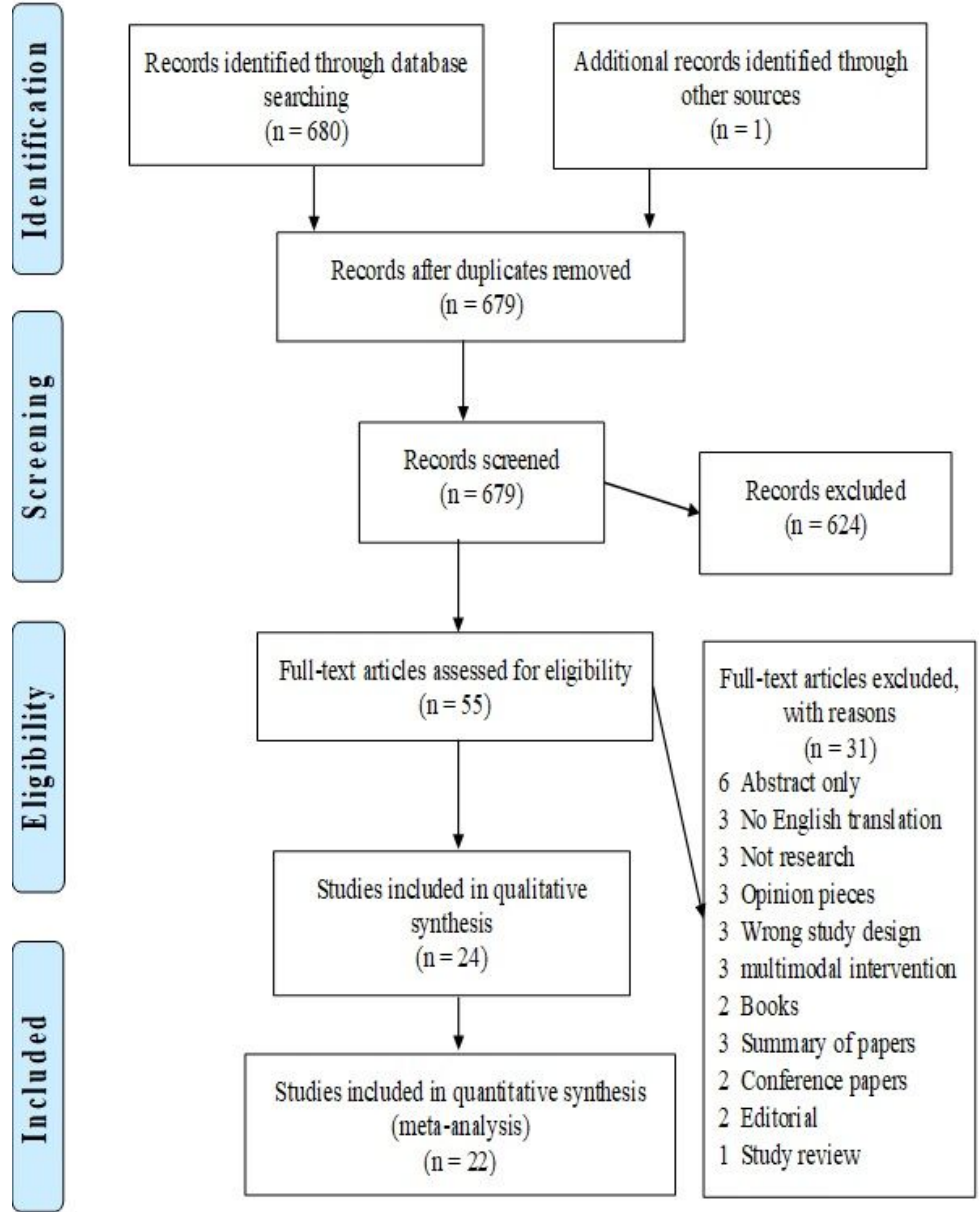

Figure 1

Prisma flow diagram (25) 


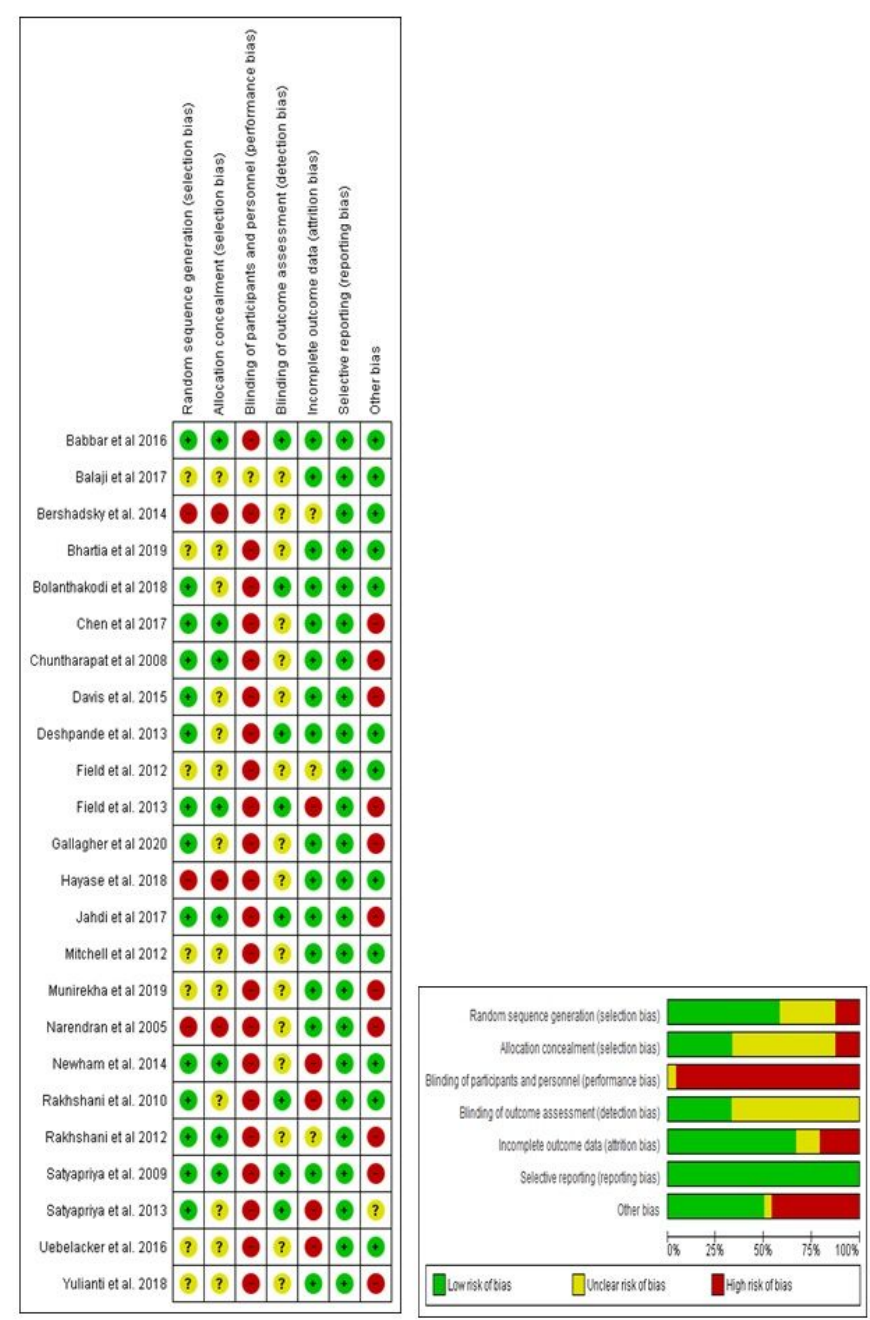

\section{Figure 2}

Summary of Risk of Bias and Risk of bias for individual studies 


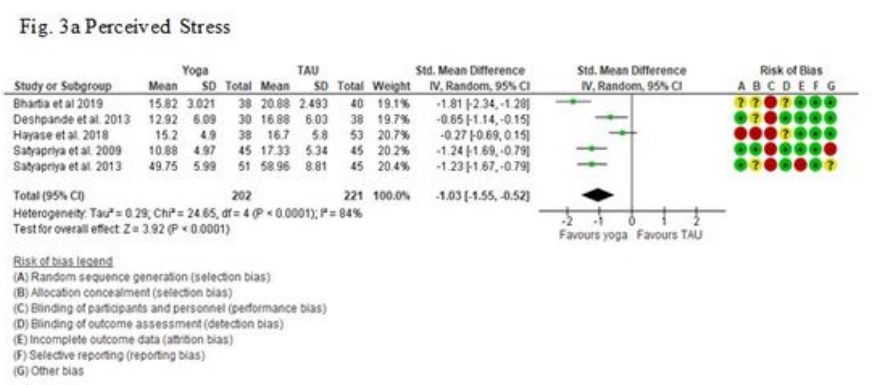

Fig 3b Perceived Stress (Excluding Hayase et al 2018)

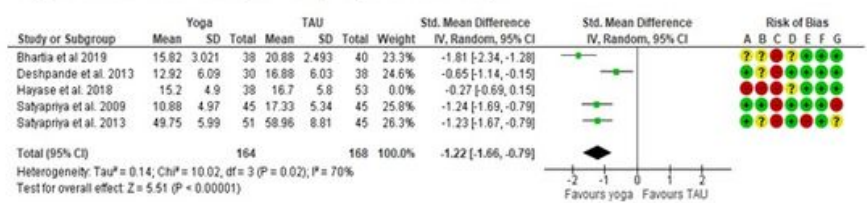

\section{Riakcetiaslesend}

(A) Random secuince generaston (selection bias)

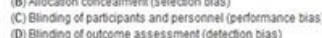

D) Binding of outceme assessment (Getection Dia

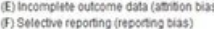

(G) Comer bias

Fig. 3c Physiological Stress

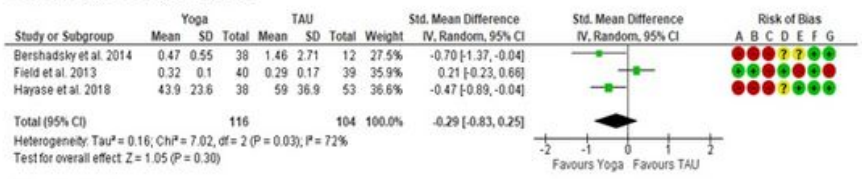

Testfor overal effect $z=1.05 p=0.30$ )

Risketriss leagent

(A) Randorm sequence generatson (seledton bias

C) Elinding of partiopants and personnel (performance bias)

(G)

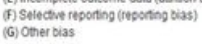

\section{Figure 3}

Primary Outcomes

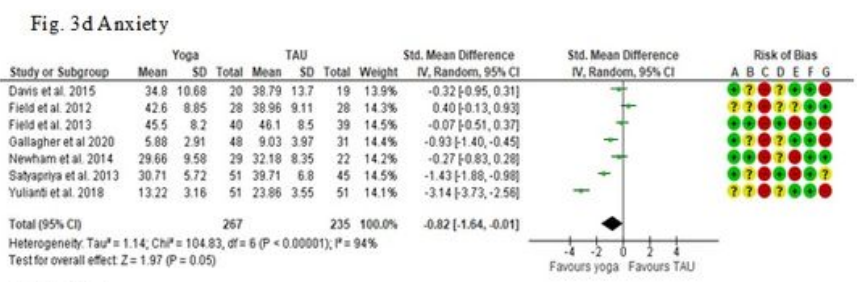

Risket bias lesend

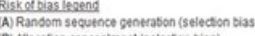

(B) Allocaston concealiment (selection bias)

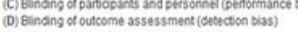

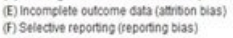

6) Othes vise

Fig. 3e Depression

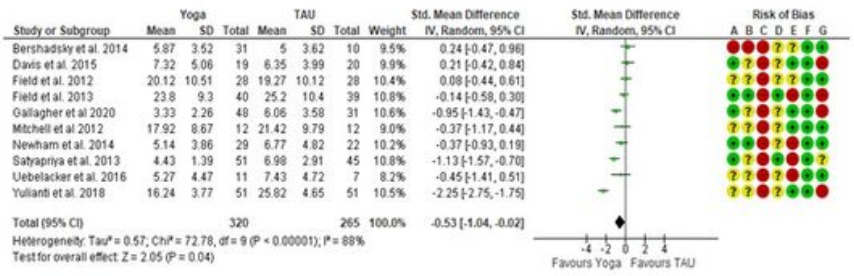

Biakefleas leatens

(A) Rasasom 5 sequencos penerason (selededon bias)

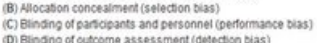

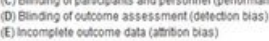

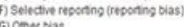




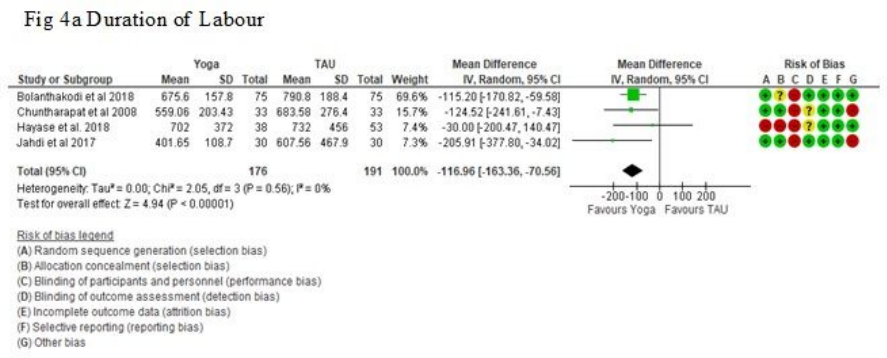

Fig. 4b Mode of Birth (Normal Vaginal Birth)

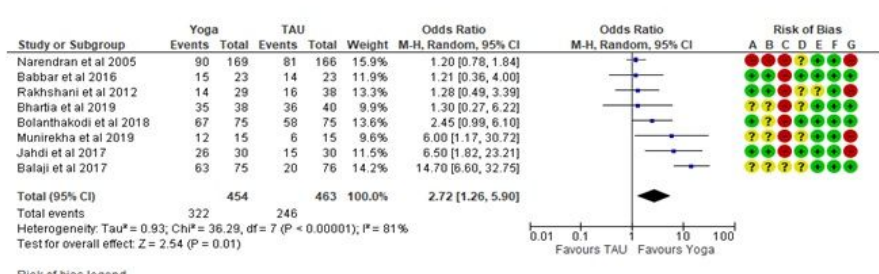

Risk of bias lesend
(A) Random sequence generatation (selection bias)

(B) Allocation concealment (selection bias)
(C) Binding of partidipants and personnel (pertormance blas)

(D) Bilinding of outcome assessment (detection bias)

(E) Incomplete outcome data (atrition bias)
(F) Selective reporting (reporting bias)
(G) Other bias

Fig. 4c Mode of Birth (Normal Vaginal Birth) excluding high risk pregnant women

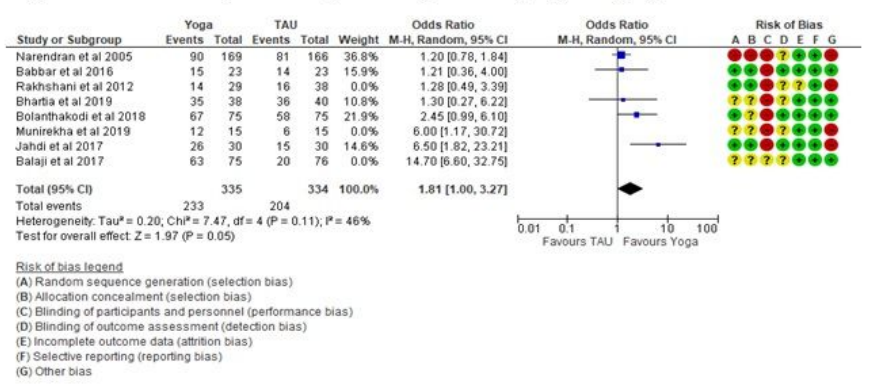

Figure 4

Secondary outcomes 

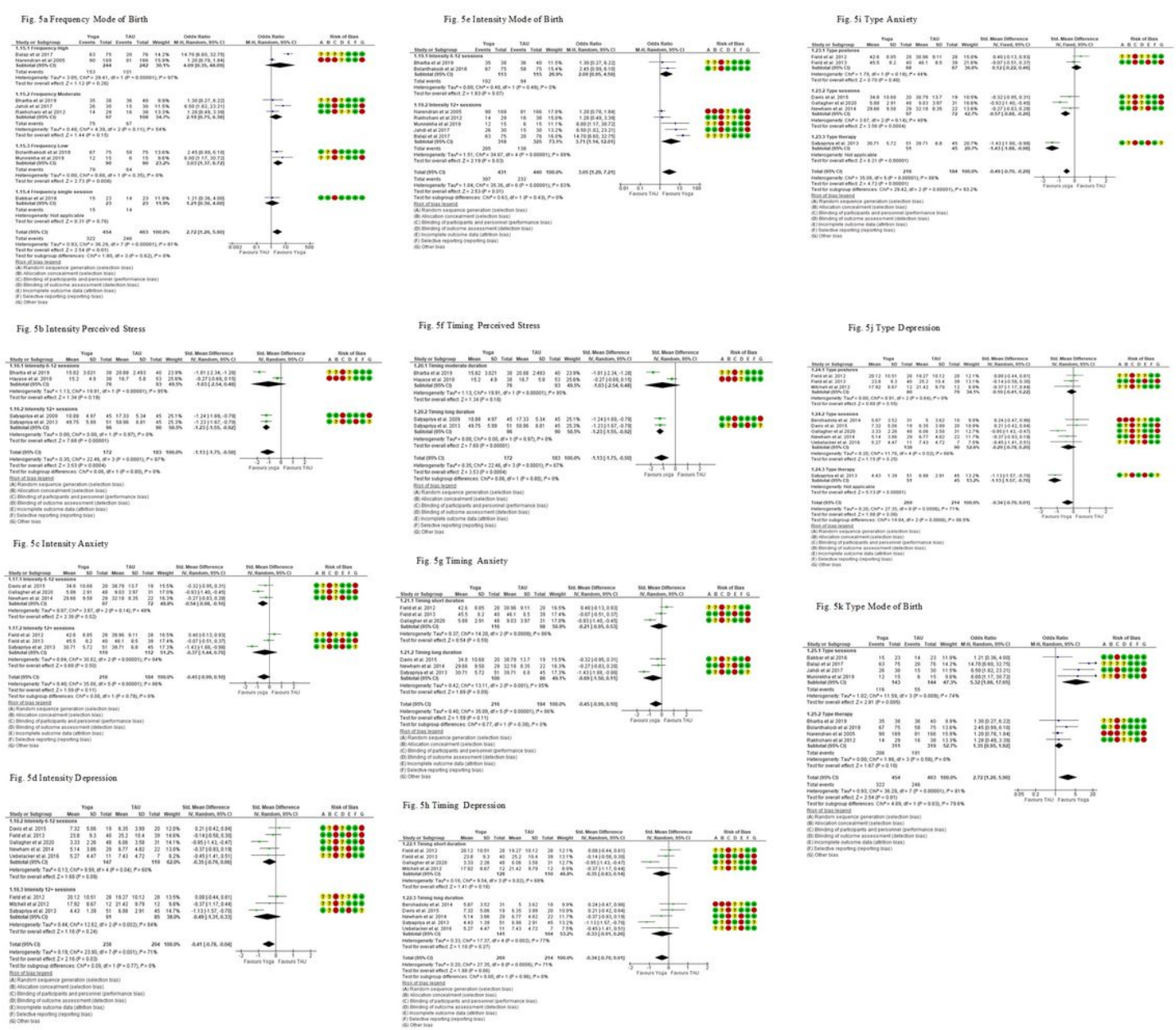

Fig. 58 Timing Anxiety
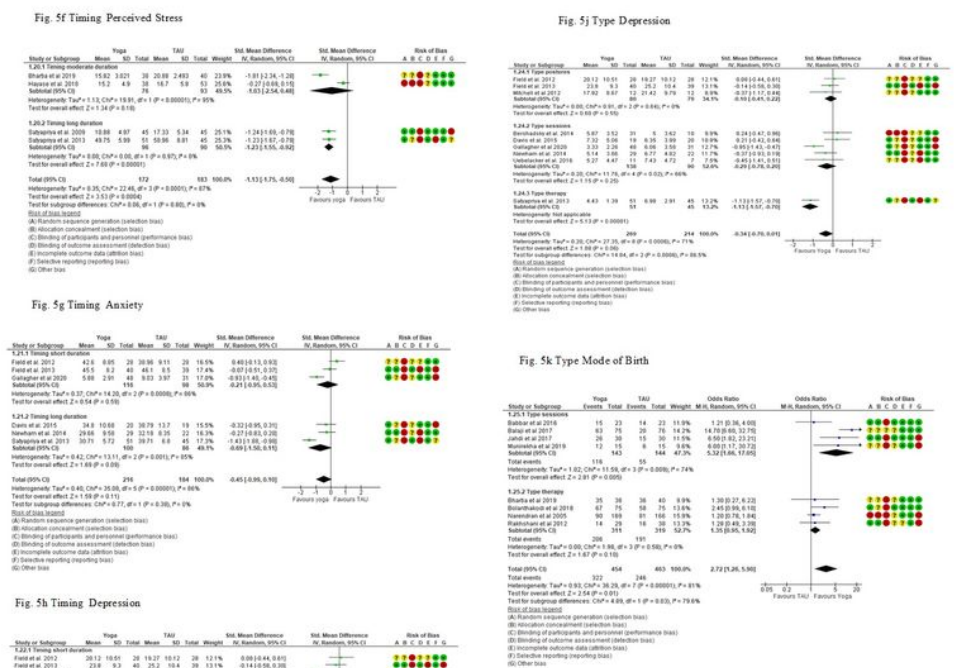

\section{Figure 5}

FITT Subgroup Analysis

\section{Supplementary Files}

This is a list of supplementary files associated with this preprint. Click to download.

- Additionalfile1PrismaChecklist.docx

- Additionalfile2EmbaseSearchStrategy.docx

- Additionalfile3Dataextractionform.docx 